
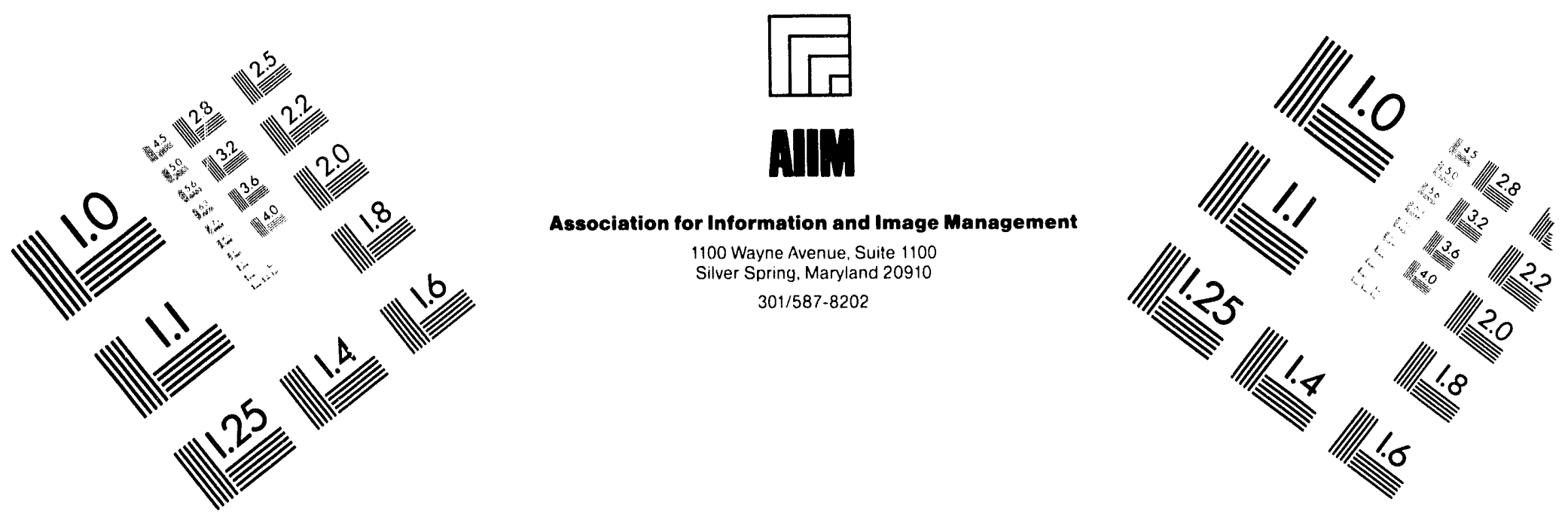

\title{
Centimeter
}

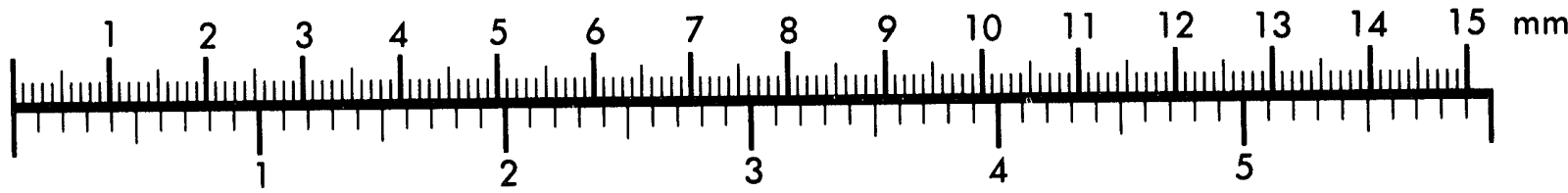

Inches
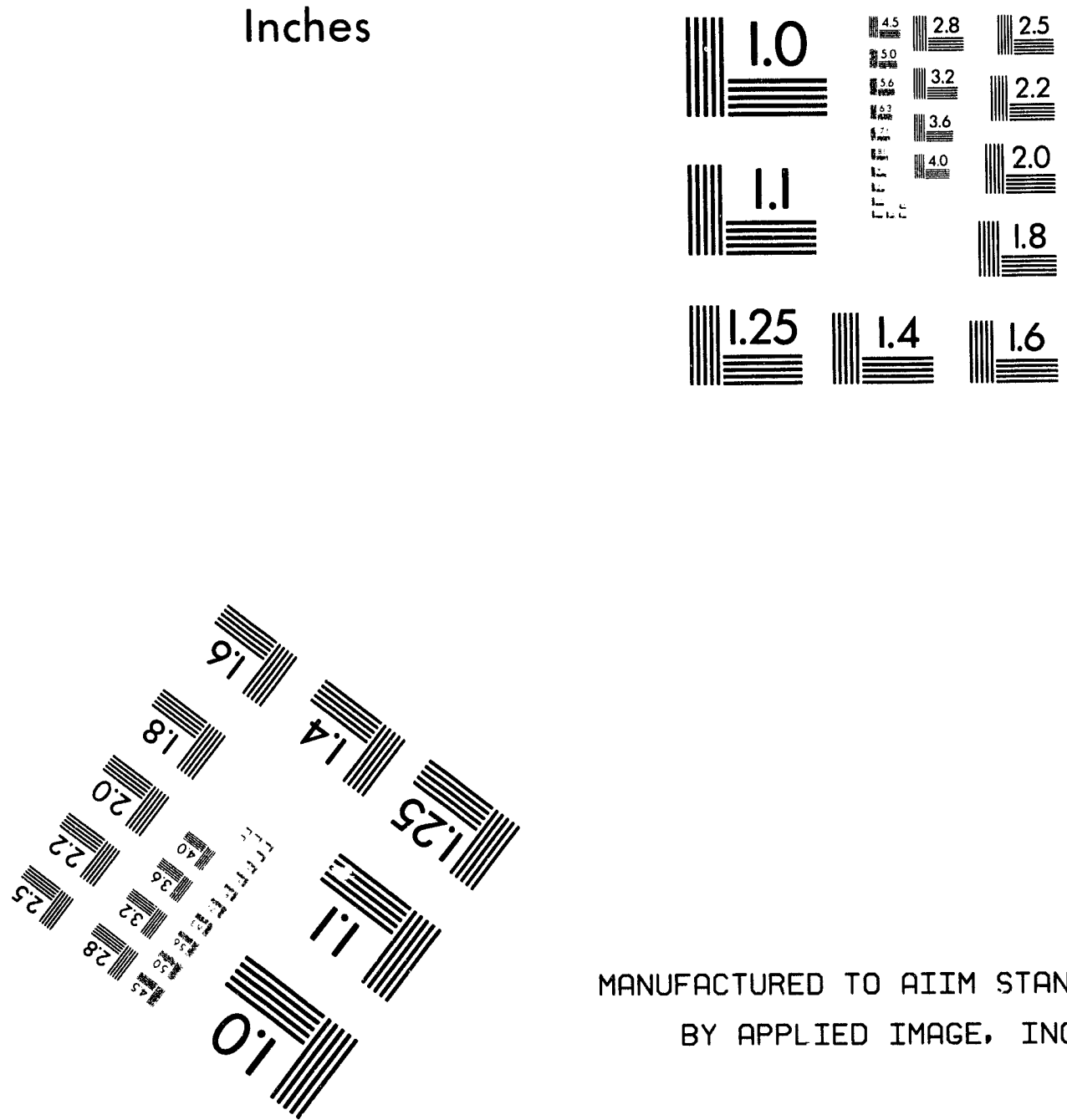

MANUFACTURED TO AIIM STANDARDS

BY APPLIED IMAGE, INC.

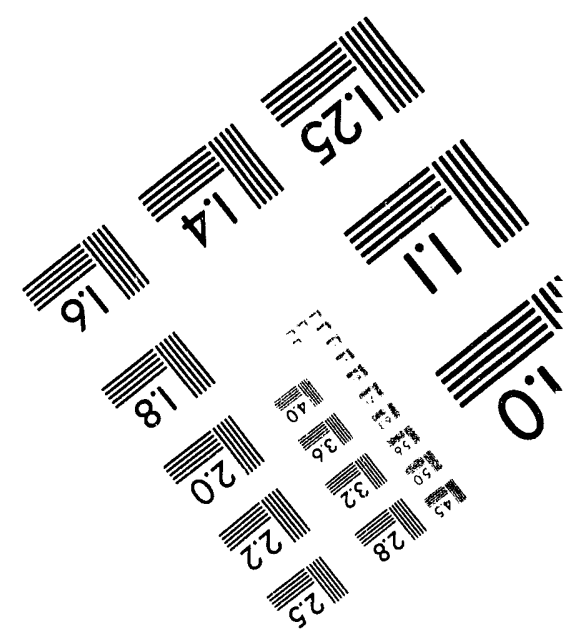



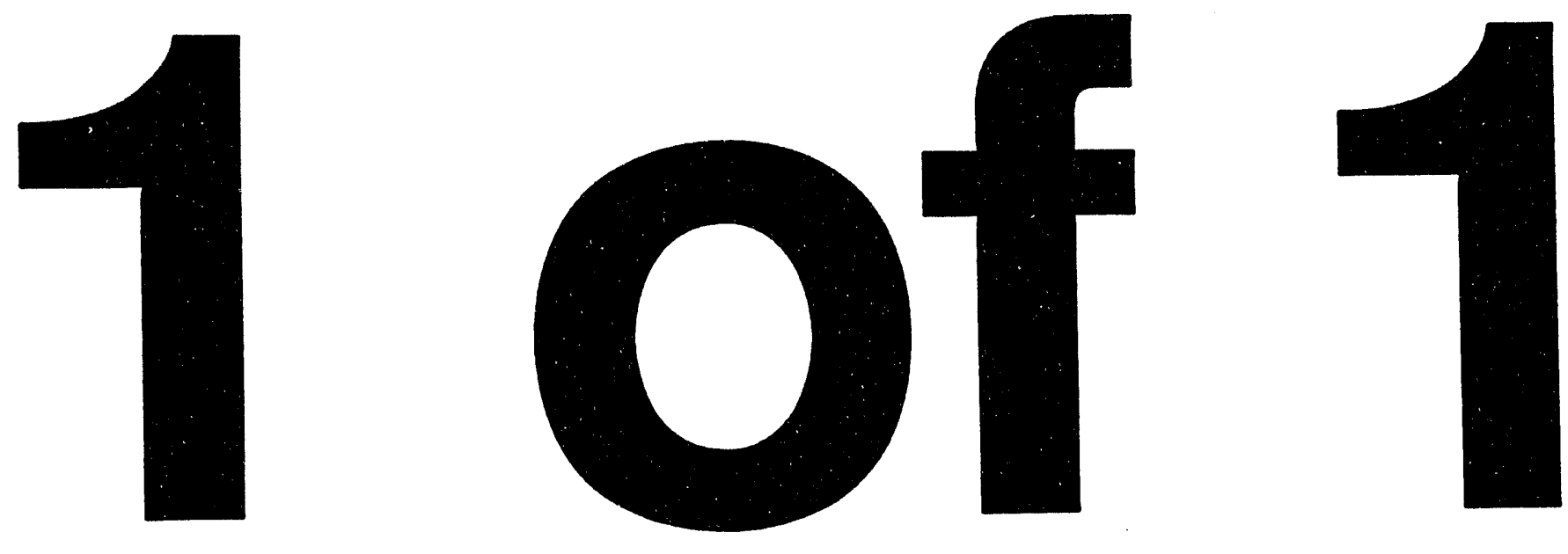
SAND 93-2274

Distribution

Unlimited Release

Category UC-706

Printed June 1994

\title{
Analysis of the Photodiode Boundary Layer Transition Indicator
}

\section{LDRD Final Report}

\author{
David W. Kuntz \\ Thermophysics Department \\ Andrew C. Wilken \\ System Design \& Engineering Department I \\ Jeffrey L. Payne \\ Aerodynamics Department \\ Sandia National Laboratories \\ Albuquerque, New Mexico 87185
}

\begin{abstract}
The photodiode transition indicator is a device which has been successfully used to determine the onset of boundary layer transition on numerous hypersonic flight vehicles. The exact source of the electromagnetic radiation detected by the photodiode at transition was not understood. In some cases early saturation of the device occurred, and the device failed to detect transition. Analyses have been performed to determine the source of the radiation producing the photodiode signal. The results of these analyses indicate that the most likely source of the radiation is blackbody emission from the heatshield material bordering the quartz window of the device. Good agreement between flight data and calculations based on this radiation source has been obtained. Analyses also indicate that the most probable source of the radiation causing early saturation is blackbody radiation from carbon particles which break away from the nosetip during the ablation process.
\end{abstract}


Page intentionally left blank. 


\section{Contents}

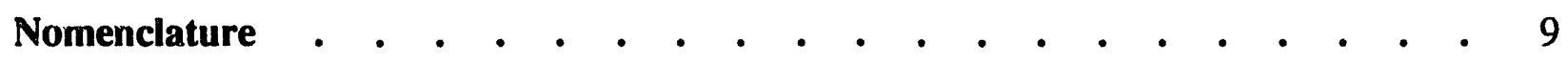

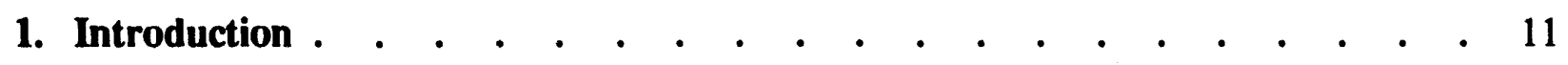

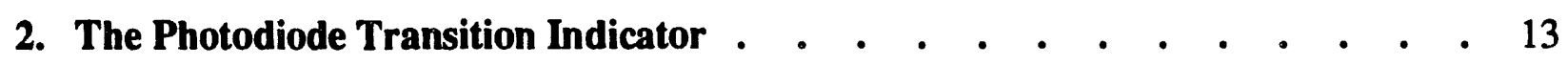

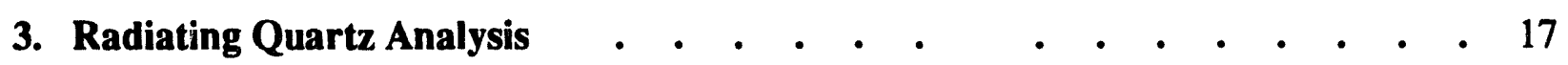

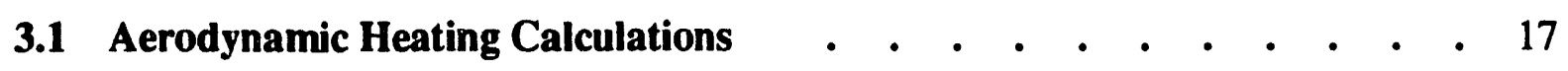

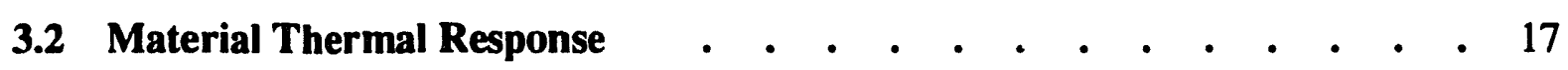

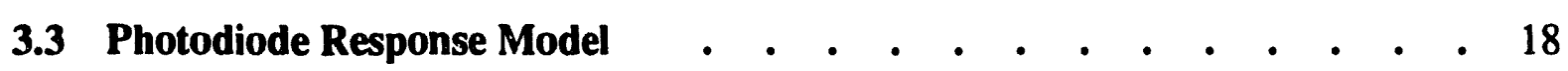

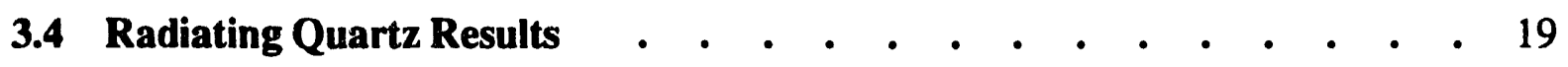

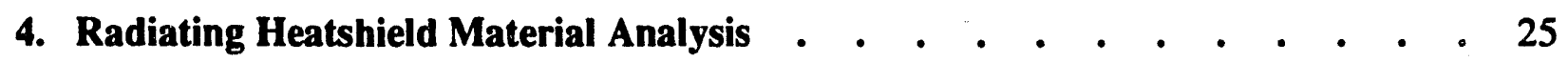

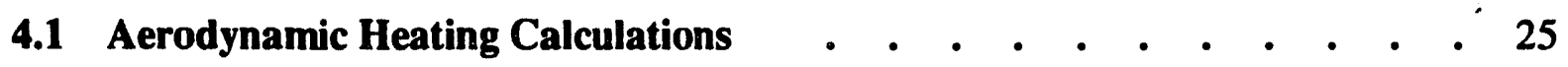

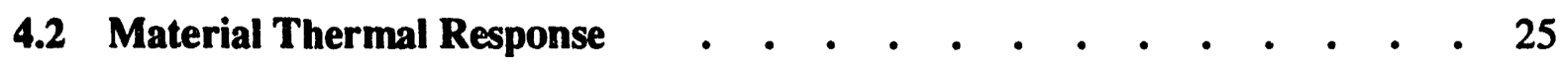

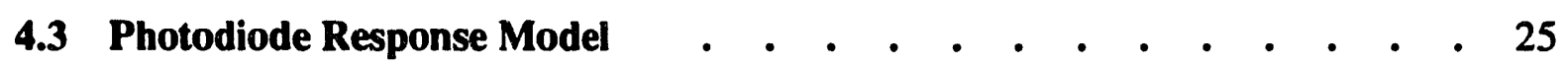

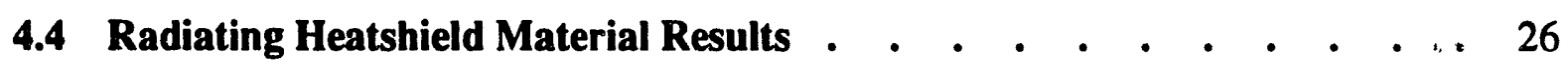

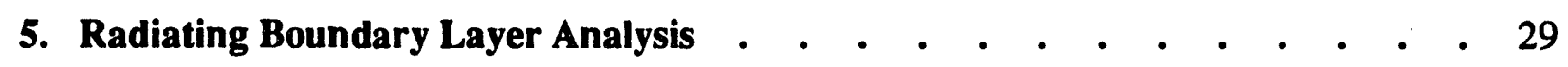

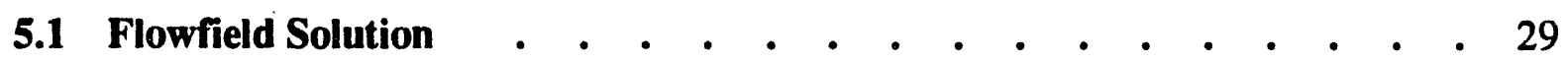

5.2 Boundary Layer Emissions • • • • • • • • • • • • • 30

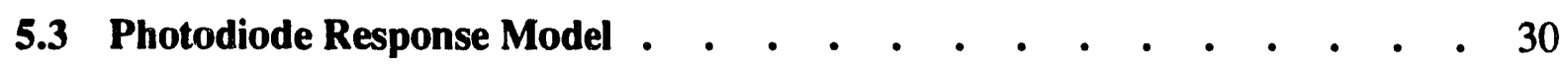

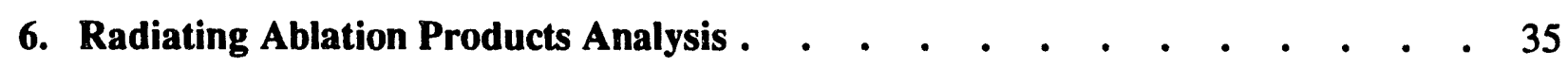

6.1 Carbon Compound Concentrations • • • • • • • • • • • • • 35

6.2 Carbon Compound Spectral Emissions • • • • • • • • • • 36

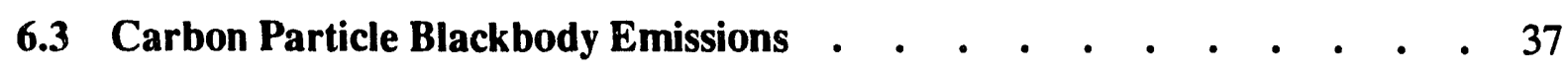


7. Summary • • • • • • • • • • • • • •

Acknowledgments $\quad$ •

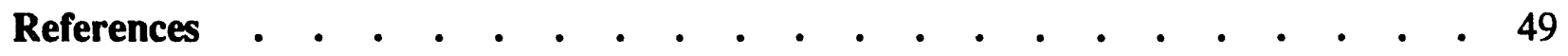

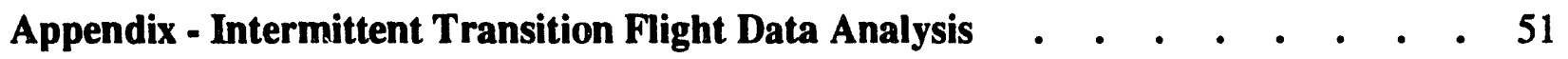




\section{Figures}

1 Photodiode transition indicator. . . . . . . . . . . . . 14

2 Photograph of photodiode flight hardware. . . . . . . . 15

3 Photodiode equivalent circuit. . . . . . . . . . . . 15

4 Photodiode signal showing boundary layer transition. . . . . . . 16

5 Photodiode signal saturating prior to boundary layer transition. . . . . 16

6 Temperature contours just prior to transition. . . . . . . . 20

7 Temperature contours $1 \mathrm{sec}$ after transition. . . . . . . . . 20

8 Temperature contours $5 \mathrm{sec}$ after transition. . . . . . . . . 21

9 Radiating quartz model. . . . . . . . . . . . . . 21

10 Quartz absorption coefficients obtained from Reference 9. . . . . . . 22

11 Quartz transmissivity obtained from Reference 9. . . . . . . . . 22

12 Photodiode spectral response. . . . . . . . . . . . . . 23

13 Radiating quartz analysis results for the photodiode indicating transition. . . 23

14 Radiating quartz analysis results for the photodiode saturating prior to transition. . . . . . . . . . . . . . . . . . 24

15 Radiating heatshield model. . . . . . . . . . . . . 27

16 Radiating heatshield analysis results for the photodiode indicating transition. . 27

17 Radiating heatshield analysis results for the photodiode saturating prior to transition. • . • . . . . . . . . . . . . . . 28

18 Calculated shock layer temperatures. . . . . . . . . . . . . 31

19 Calculated laminar mass fractions. . . . . . . . . . . 32

20 Calculated laminar ion mass fractions. . . . . . . . . . . 32

21 Calculated turbulent mass fractions. . . . . . . . . . . 33 
22 Calculated turbulent ion mass fractions. . . . . . . . . . . . 33

23 Calculated laminar spectrum.

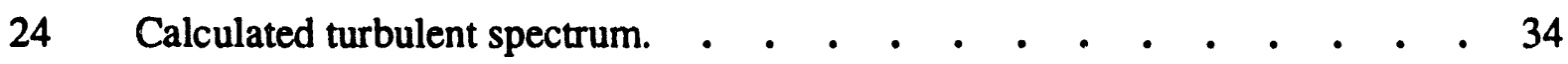

25 Calculated laminar carbon mass fractions. $\quad$ • . . . . . . . 39

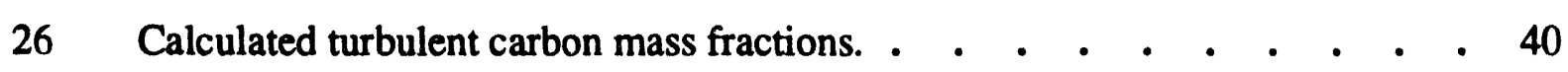

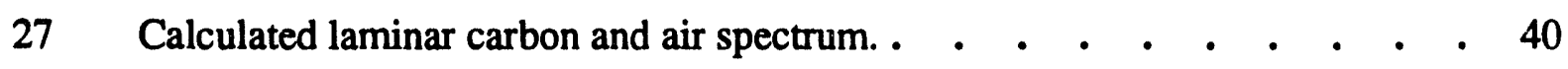

28 Calculated turbulent carbon and air spectrum. . . . . . . . . . 41

29 Comparison of photodiode spectral power. $\quad$ - . . . . . . . . 41

30 Comparison of photodiode spectral current. . . . . . . . . . . 42

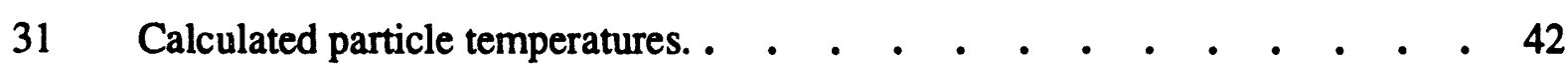

32 Calculated photodiode signal resulting from carbon particles. . • • . . 43

A1 Co-located photodiode and near-surface thermocouple indicating intermittent transition.. . . . . . . . . . . . . . 52

A2 Cold wall heat transfer rate used to simulate intermittent transition. . . . . 53

A3 Comparison of flight data and analysis results for the intermittent transition case. . . . . . . . . . . . . . . . 53 


\section{Nomenclature}

\begin{tabular}{|c|c|}
\hline$A_{\text {hsr }}$ & Area of a heatshield region \\
\hline$A_{q d}$ & Area of a quartz disk \\
\hline$A_{\text {ws }}$ & Area of the window surface \\
\hline e & Electronic charge \\
\hline $\mathrm{E}_{\mathrm{B}, \lambda}$ & Spectral blackbody emissive power \\
\hline $\mathrm{F}_{\text {hst-pd }}$ & View factor from a heatshield region to the photodiode \\
\hline$F_{\text {qd-pd }}$ & View factor from a quartz disk to the photodiode \\
\hline$F_{\text {ws-pd }}$ & View factor from the window surface to the photodiode \\
\hline $\mathrm{I}_{\mathrm{L}}$ & Photodiode photogenerated current \\
\hline Io & Photodiode reverse leakage current \\
\hline $\mathbf{I}_{\mathbf{T}}$ & Photodiode terminal current \\
\hline $\mathbf{I}_{\mathrm{T}, \mathrm{hsr}}$ & $\begin{array}{l}\text { Photodiode terminal current resulting from the emissions } \\
\text { of a heatshield region }\end{array}$ \\
\hline $\mathrm{I}_{\mathrm{T}, \mathrm{qd}}$ & $\begin{array}{l}\text { Photodiode terminal current resulting from the emissions } \\
\text { of a single quartz disk }\end{array}$ \\
\hline k & Boltzmann's constant \\
\hline $\mathbf{K}$ & Maximum theoretical transmittance \\
\hline L & Flight vehicle length \\
\hline $\mathbf{M}_{\infty}$ & Freestream Mach number \\
\hline $\mathbf{n}$ & Index of refraction \\
\hline $\mathrm{r}^{*}$ & $\begin{array}{l}\text { Distance from vehicle surface, divided by the distance } \\
\text { from the surface to the shock wave }\end{array}$ \\
\hline
\end{tabular}


$\mathbf{R}$

$\mathrm{R}_{\mathrm{L}}$

$\mathrm{R}_{\mathrm{pd}, \lambda}$

$\mathbf{R}_{\mathbf{S}}$

$S_{\lambda}$

$t_{q d}$

$\mathrm{T}$

$\mathrm{T}_{\mathbf{p}}$

$\mathrm{T}_{\text {ve }}$

$V_{d}$

$\mathrm{V}_{\mathrm{L}}$

$\mathbf{x}$

$\alpha_{\lambda, T}$

$\varepsilon$

$\varepsilon_{\lambda, T}$

$\lambda$

$\tau_{\lambda, T}$
Single surface reflection loss

Load resistance

Photodiode spectral response

Photodiode series resistance

Spectral power

Thickness of a quartz disk

Temperature

Carbon particle temperature

Vibrational-electron-electronic temperature

Photodiode voltage

Load voltage

Axial position on the flight vehicle

Spectral absorption coefficient

Total emissivity

Spectral emissivity

Wavelength

Spectral transmissivity 


\section{Introduction}

Boundary layer transition, the change of the fundamental nature of a boundary layer from a laminar to a turbulent state, is one of the most critical phenomenon in high speed flight. As a boundary layer changes from laminar to turbulent, the drag on the vehicle increases significantly, and the surface heating rises by as much as a factor of 10 . The successful operation of high speed vehicles such as the National Aerospace Plane (NASP) may depend on the ability to sense the boundary layer state and adjust the flight characteristics of the vehicle to avoid turbulent boundary layer flow. The photodiode transition indicator is a device which was developed at Sandia National Laboratories for the purpose of determining the local condition of the boundary layer on a hypersonic flight vehicle ${ }^{1}$. The device receives an optical signal through a quartz window in the vehicle surface and senses an increase in light intensity which is directly indicative of turbulent flow. Flight tests have shown that, at transition, the photodiode produces a significant step increase in signal strength. However, the specific cause of this increased optical signal was . not known. In recent flight tests, several of these devices have saturated prior to boundary layer transition and thus failed to provide useful data.

Analyses have been conducted to determine the exact cause of the photodiode signal, and to develop a technique for predicting the diode signal strength for given flight conditions. The results of these analyses are reported in this paper. 
Page intentionally left blank. 


\section{The Photodiode Transition Indicator}

A diagram of the photodiode transition indicator is shown in Figure 1 and a photograph of photodiode flight hardware is shown in Figure 2. As illustrated in Figure 1, a conical quartz window is mounted flush with the surface of the heatshield and is held in place by a thin layer of epoxy. A silicon or gallium arsenide photodiode is mounted beneath the window such that electromagnetic radiation emitted by external sources, the quartz window material, or the heatshield material surface bordering the window material, is incident on the photodiode surface.

The electrical circuit connected to the photodiode is illustrated in Figure 3 . The leads from the photodiode are connected to a load resistor, $R_{L}$, and the voltage across this resistor, $V_{L}$, forms the signal used to determine boundary layer transition. The terminal current, $\mathrm{I}_{\mathrm{T}}$, can be determined from the expression ${ }^{1}$ :

$$
I_{T}=I_{L}-I_{O}\left[\exp \left(\frac{e V_{d}}{k T}\right)-1\right]
$$

In this equation, $I_{L}$ is the photogenerated current, $I_{O}$ is the photodiode reverse leakage current, $e$ is the electronic charge, $V_{d}$ is the photodiode voltage, $k$ is Boltzmann's constant, and $T$ is the temperature. The photodiodes discussed in this paper are operated in what is known as the linear response mode, such that the load resistance is relatively small and subsequently the photodiode voltage is small. This mode of operation results in the exponential term approaching a value of one in the above equation, which then simplifies to:

$$
\mathbf{I}_{\mathrm{T}} \cong \mathrm{I}_{\mathrm{L}}
$$

Thus the current through the load resistor is approximately linearly proportional to the photon flux on the photodiode surface.

For a properly functioning photodiode transition indicator, the load voltage $\left(V_{L}\right)$ rises extremely rapidly at boundary layer transition. This is illustrated in Figure 4, which shows the load voltage, plotted in percent of the telemetry channel full scale, as a function of time measured from an arbitrary reference. The near instantaneous rise in voltage as boundary layer transition passes over the quartz window is visible at a time of approximately 15 seconds. The situation in which the load voltage saturates the telemetry channel prior to transition is illustrated in Figure 5, in which the voltage rises smoothly to the full scale limit, without the characteristic rapid rise associated with transition. In the case of the data presented in Figure 5, the fact that transition occurred after the photodiode saturated was confirmed with thermocouple data.

The data presented in Figures 4 and 5 was obtained from photodiodes mounted on a ballistic reentry vehicle equipped with a carbon-carbon nosetip and a carbon-phenolic heatshield. The photodiode which produced the signal presented in Figure 4 was located at an axial position near the center of the vehicle, and the photodiode which produced the signal presented in Figure 5 was located at an axial position significantly farther forward on the vehicle. As part of the flight experiment, this vehicle experienced a rapid deceleration near the end of the flight, which resulted 
in the signal from the center photodiode returning to a near zero value. This signal drop-off will be presented later in this paper with analysis results. Unfortunately, the forward photodiode failed during the deceleration process, and thus produced no data during the signal drop-off.

In order to specify the circuit parameters and telemetry channel limits required to prevent diode saturation prior to boundary layer transition, it is necessary to be able to model the characteristics of the photodiode transition irdicator. This model development effort depends upon a clear understanding of the source of the electromagnetic radiation causing the signal. In order to determine the source of the radiation, and to develop a model of the photodiode transition indicator, detailed analyses of the device were performed. Each of these analyses was directed towards determining the likelihood that a proposed radiation source was responsible for the photodiode transition signal. Four proposed radiation sources were investigated: 1) radiation from the quartz window material, 2) radiation from the heatshield material bordering the quartz window, 3) radiative emissions from the gases within the boundary and shock layers, and 4) radiation from ablation products. The results of these analyses are summarized in the sections which follow.

\section{External Flow Direction}

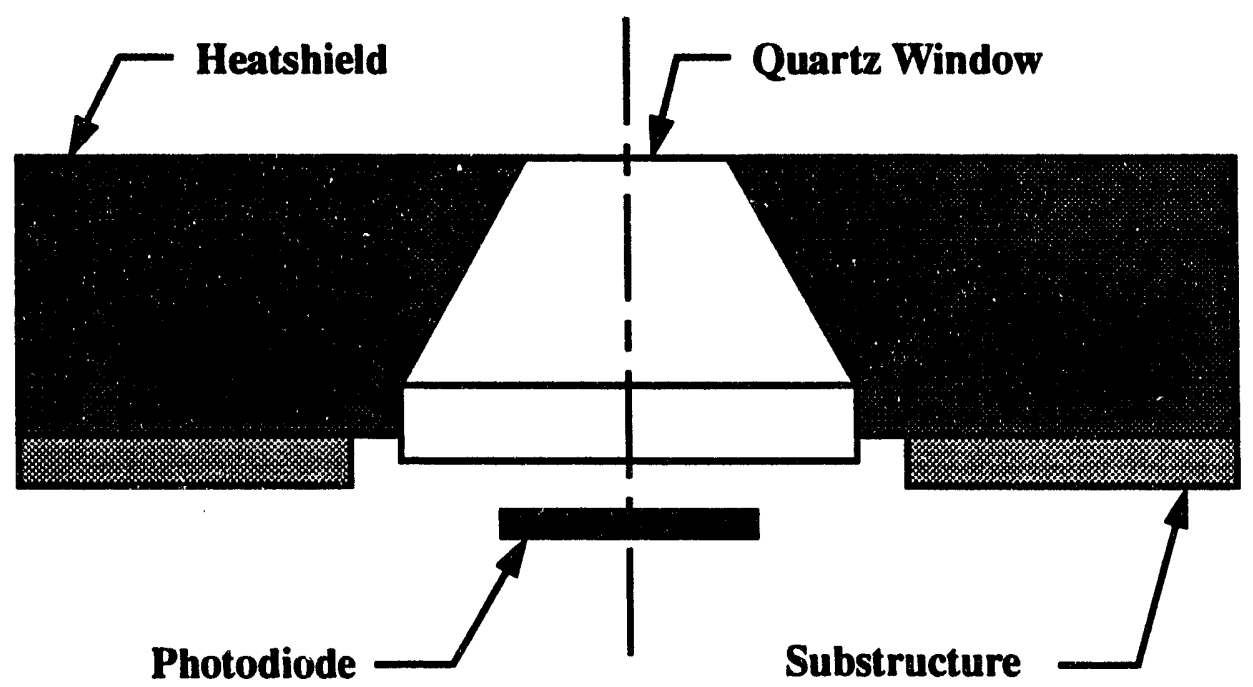

Figure 1: Photodiode transition indicator. 


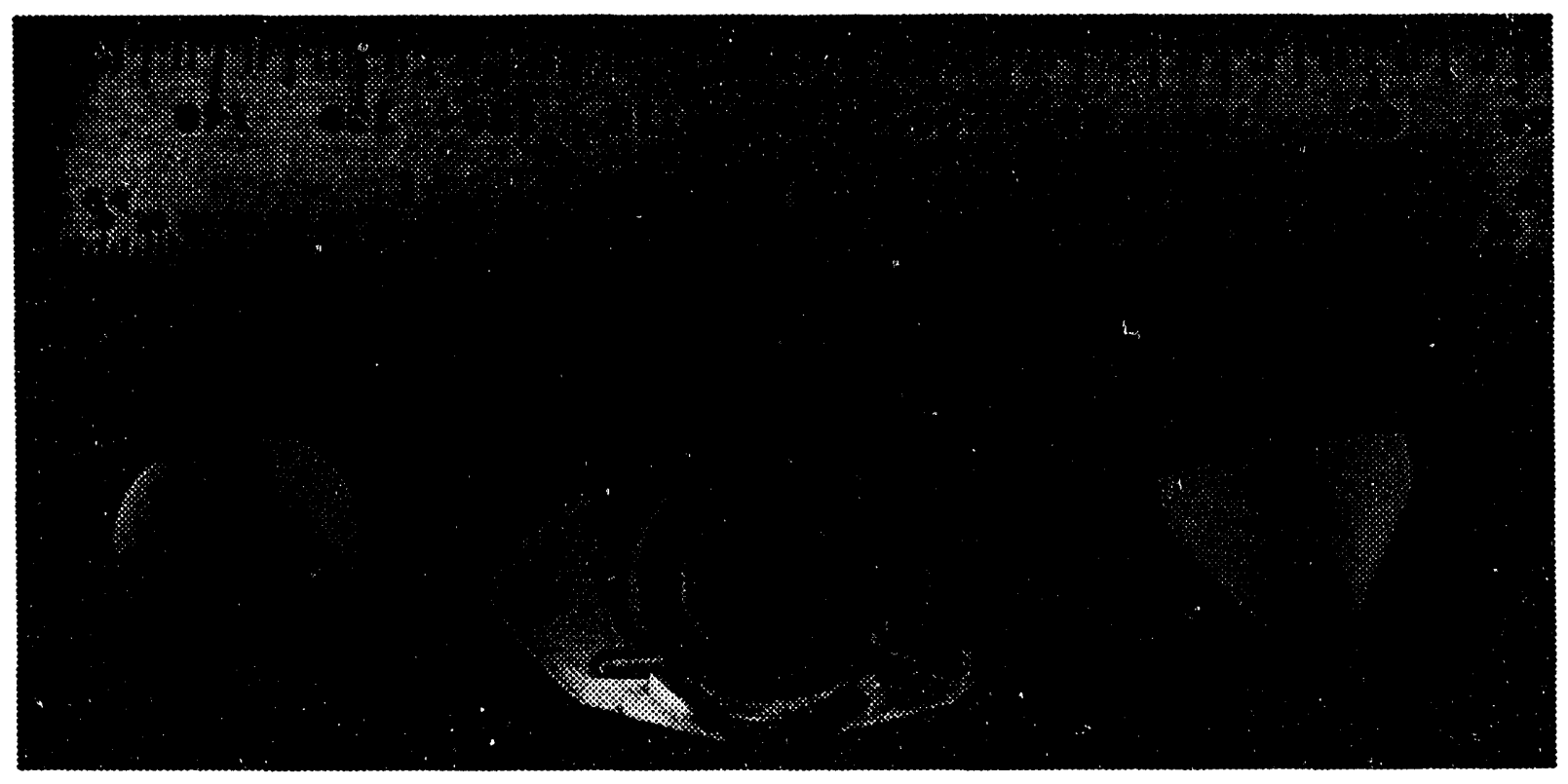

Figure 2: Photograph of photodiode flight hardware.

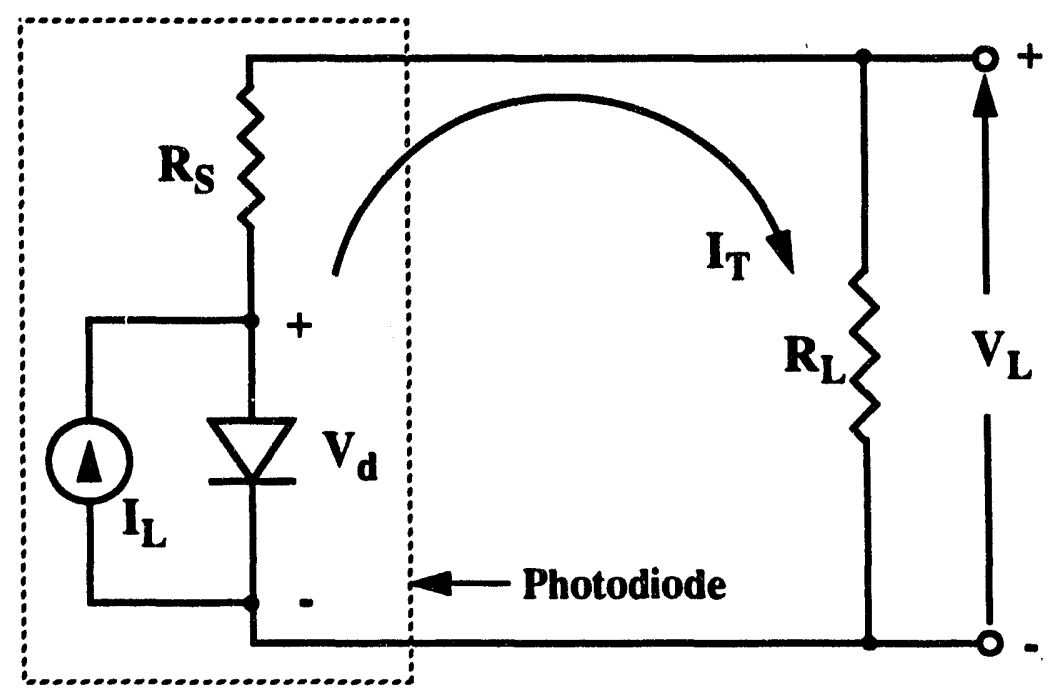

Figure 3: Photodiode equivalent circuit. 


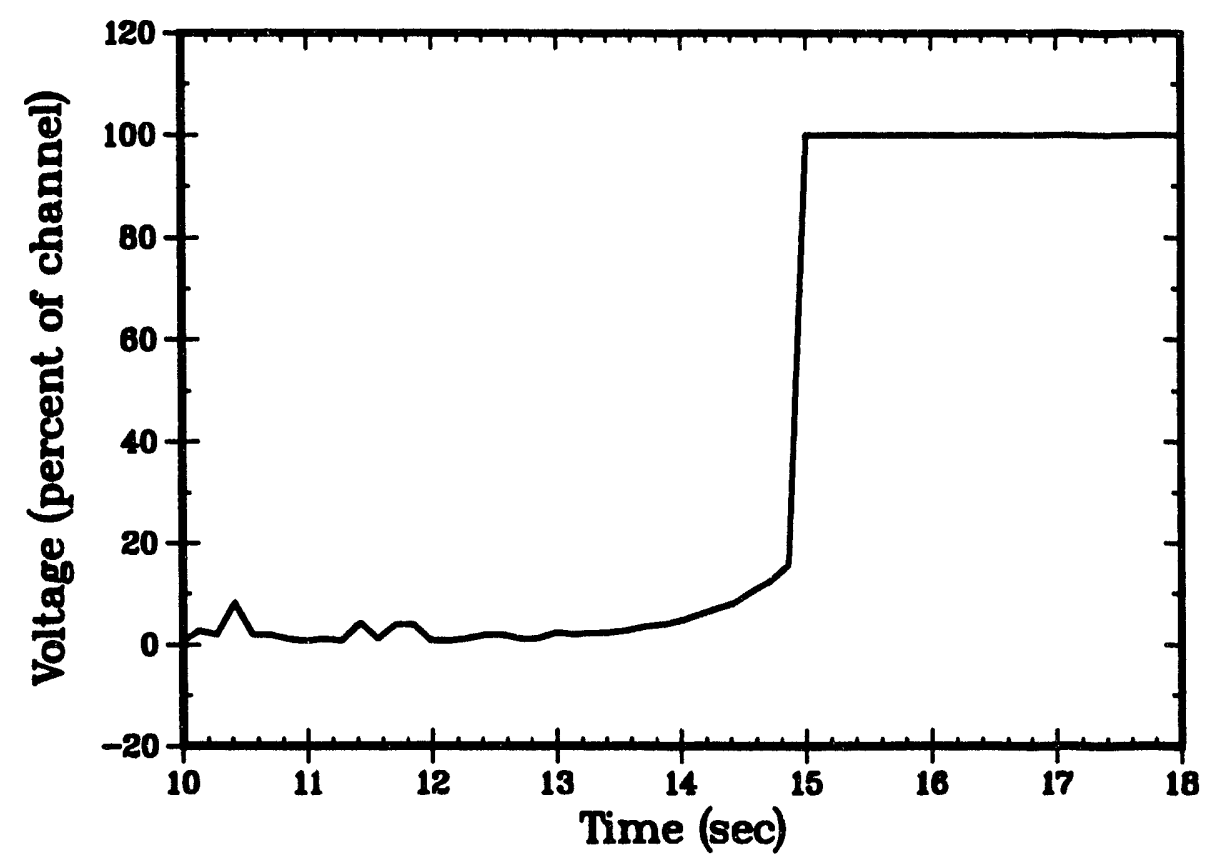

Figure 4: Photodiode signal showing boundary layer transition.

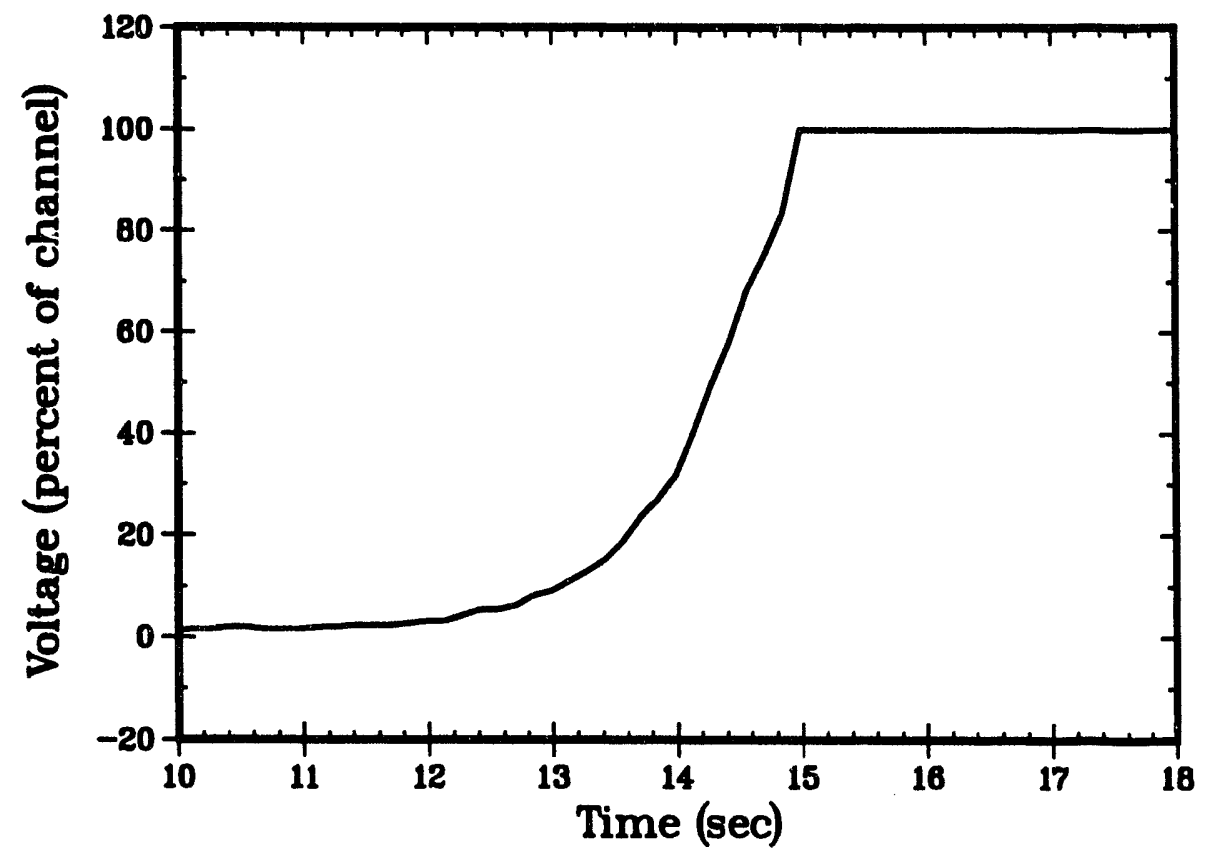

Figure 5: Photodiode signal saturating prior to boundary layer transition. 


\section{Radiating Quartz Analysis}

The source of the photodiode transition signal originally proposed in Reference 1 involves radiation from the quartz window material. The temperature of the surface of the quartz window rises rapidly at boundary layer transition due to the near instantaneous rise in heating rate. It was proposed that the associated rapid rise in temperature resulted in the quartz material emitting in a portion of the electromagnetic spectrum which is within the spectral response of the photodiode. In order to determine whether or not the quartz was the source of the radiation sensed by the photodiode at transition, a detailed analysis of the quartz window material and the resulting photodiode response was performed. The analysis was performed using the geometry and flight conditions for the center photodiode which produced the signal shown in Figure 4.

\subsection{Aerodynamic Heating Calculations}

The aerodynamic heating on the quartz window and surrounding heatshield material was calculated with the GE2IT ${ }^{2}$-SANDIAC ${ }^{3}-$ HIBLARG $^{4}$ family of computer programs for the high velocity portion of the flight $\left(M_{\infty}>2\right)$, and with the LOVEL 5 computer program for the low velocity portion of the flight $\left(M_{\infty} \leq 2\right)$. GE2IT solves for the inviscid flowfield in the vicinity of the spherical nosetip, SANDIAC solves for the inviscid flowfield over the conical portion of the body, and HIBLARG solves the integral boundary layer equations over the surface in order to determine the cold-wall heating rates and boundary layer edge conditions for both laminar and turbulent flow. LOVEL uses analytical and empirical relations to determine cold-wall heating rates and boundary layer edge conditions on simple geometries at relatively low Mach numbers. The boundary layer transition time was set equal to that obtained on the flight vehicle at the photodiode locations. The GE2IT-SANDIAC-HIBLARG codes predicted an increase in coldwall heating rate of a factor of 6.2 at boundary layer transition. This increase was assumed to occur over a time span of 0.1 seconds.

\subsection{Material Thermal Response}

The conical shape of the quartz window and the need to predict the quartz temperature distribution as accurately as possible resulted in the need to perform a two-dimensional axisymmetric material response analysis of the quartz and surrounding heatshield material. For this analysis, the $\mathrm{FCV}^{6}$ computer program, currently under development at Sandia National Laboratories, was used. This code uses a moving-mesh algorithm to model ablating twodimensional geometries utilizing either a heat of formation and melt temperature ablation model or a specified surface recession rate and surface temperature boundary condition.

At the time this analysis was performed, FCV was not capable of solving a problem with two different ablating materials. Thus, the specified surface recession rate and surface temperature boundary condition was used, and the surface recession rates and the surface temperatures for the quartz and the heatshield material were determined using the one-dimensional ablation-thermal response program SODDIT ${ }^{7}$. Within this program, the heat of formation and melt temperature ablation model was used to determine the necessary boundary layer conditions for the quartz and heatshield materials for the FCV calculations.

The results of the FCV calculations for the quartz window and the surrounding heatshield material just prior to boundary layer transition, one second after boundary layer transition, and 
five seconds after boundary layer transition, are shown in Figures 6, 7, and 8, respectively. These figures show both the computational grid and the temperature contours. The left side of each figure is the window centerline, and the edge of the quartz window can be distinguished by the very thin nodes between the quartz and the heatshield material which model the epoxy layer. At approximately one second after boundary layer transition, the surface of the quartz has reached its melt temperature of $2,333 \mathrm{~K}\left(4,200^{\circ} \mathrm{R}\right)$ and surface recession of this material begins. At five seconds after transition, a significant amount of ablation has occurred in both materials. It should be noted that the rounding of the heatshield material at the surface near the quartz-heatshield junction was artificially implemented. The surface recession rates were obtained from onedimensional solutions, and thus formed a sharp edge at the material junction. This was not considered realistic, and thus the recession rates for the material near the junction were smoothed between the quartz and heatshicld values to form the smooth junction shown in Figure 8.

\subsection{Photodiode Response Model}

The radiative emission of the quartz was modeled by dividing the window into a series of thin layers which were treated as disks radiating to the photodiode surface. This is illustrated in Figure 9. Each thin layer of quartz was assumed to be at a uniform temperature which was calculated from the radial temperature distribution obtained from the FCV solution with an areaaveraging technique.

The current produced by the photodiode at each time step as a result of the radiation emitted by a single quartz disk can be determined from the relation:

$$
I_{T, q d}=\int\left(A_{q d} F_{q d-p d} \varepsilon_{\lambda, T} E_{B, \lambda} \tau_{\lambda, T} R_{p d, \lambda}\right) d \lambda
$$

In this equation $A_{q d}$ is the area of the isothermal quartz disk, $F_{q d-p d}$ is the view factor from the quartz disk to the photodiode (obtained from Reference 8), $\varepsilon_{\lambda, \mathrm{T}}$ is the spectral emissivity of the quartz disk, $\mathrm{E}_{\mathrm{B}, \lambda}$ is the spectral emissive power of a blackbody radiator at the same temperature as the quartz disk, $\tau_{\lambda, \mathrm{T}}$ is the total transmissivity of the quartz between the radiating disk and the photodiode, including a single surface reflective loss, and $R_{p d, \lambda}$ is the spectral response curve of the photodiode.

The spectral emissivity of the quartz disk is obtained from the relation:

$$
\varepsilon_{\lambda, T}=1-\exp \left(-\alpha_{\lambda, T t_{q d}}\right)
$$

In this relation $t_{\mathrm{qd}}$ is the thickness of the quartz disk, and $\alpha_{\lambda, \mathrm{T}}$ is the absorption coefficient for the window material. The absorption coefficient is both a function of wavelength and temperature. High temperature absorption coefficient data for Dynasil, the quartz material flown on the reentry vehicle, was not available, and thus data for a similar material, General Electric Type 151, obtained from Reference 9, was used. Linear extrapolation in temperature was necessary to estimate absorption coefficients near the quartz melt temperature. The absorption coefficient values and resultant transmissivity values are shown in Figures 10 and 11 , respectively. 
The total transmissivity of the quartz between the radiating disk and the photodiode, $\tau_{\lambda, T}$, was obtained from the relation:

$$
\tau_{\lambda, T}=K \prod\left[\exp \left(-\alpha_{\lambda, T} t_{q d}\right)\right]
$$

Here, the product is taken over all the quartz disks between the disk of interest and the backface of the window. The quantity $\mathrm{K}$ is the maximum theoretical transmittance and is obtained from the relation:

$$
\mathbf{K}=(1-\mathbf{R})
$$

where $R$ is the single surface reflection loss obtained from the index of refraction $\mathrm{n}$ from the relation:

$$
R=\left[\frac{n-1}{n+1}\right]^{2}
$$

The spectral response curve of the silicon photodiode, $R_{p d, \lambda}$, was obtained experimentally at Sandia National Laboratories, and is presented in Figure 12. The photodiode has a sensitivity range from wavelengths of approximately $350 \mathrm{~nm}$ to $1200 \mathrm{~nm}$. Thus, in this analysis, integrations over wavelength were performed between these limits.

The total current emitted by the photodiode at a given time is determined by summing the contributions from each quartz disk, thus including the effects of radiation emitted from within the entire quartz window. The load voltage is then determined by taking the product of the total current and the resistance of the load resistor. As mentioned above, the photodiodes are operated in the linear mode, such that the current is not a function of the size of the load resistor over the range of interest.

\subsection{Radiating Quartz Results}

The results of the radiating quartz model analysis are compared with flight data in Figures 13 and 14. The flight data is the same as that presented in Figures 4 and 5, respectively. As mentioned previously, this reentry vehicle experienced a rapid deceleration after boundary layer transition, and the effects of this deceleration can be seen in the flight data of Figure 13, where the photodiode signal drops between a time of 25 and 30 seconds.

The results shown in Figures 13 and 14 indicate that, although the quartz does emit at a level which can be detected by the photodiode, the radiation within the spectral response of the photodiode is not sufficient to generate the signal observed at boundary layer transition. This result is primarily due to the relatively low emissivity of the quartz material in the wavelength band which is visible to the photodiode. Thus, an additional source of radiation must be present to generate the measured signal. 

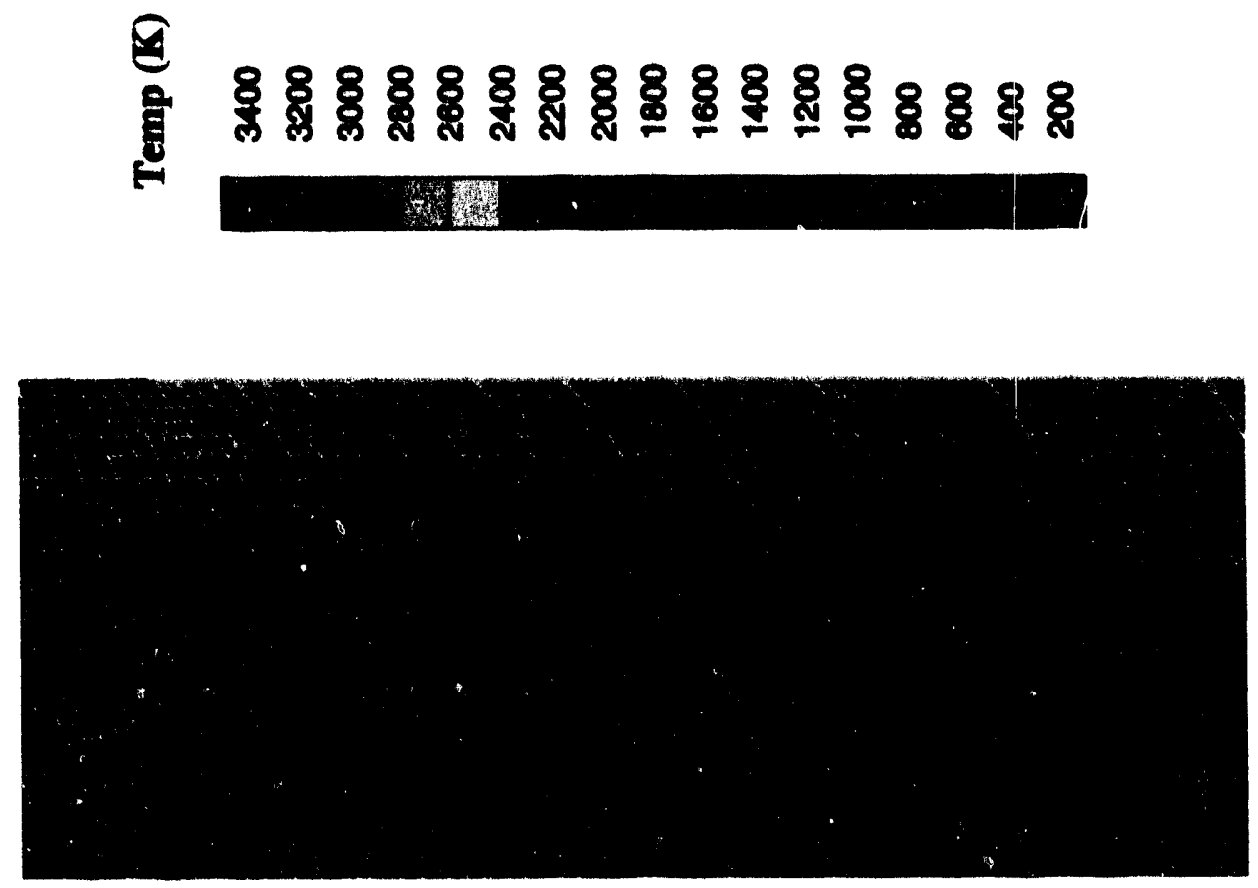

Figure 6: Temperature contours just prior to transition.
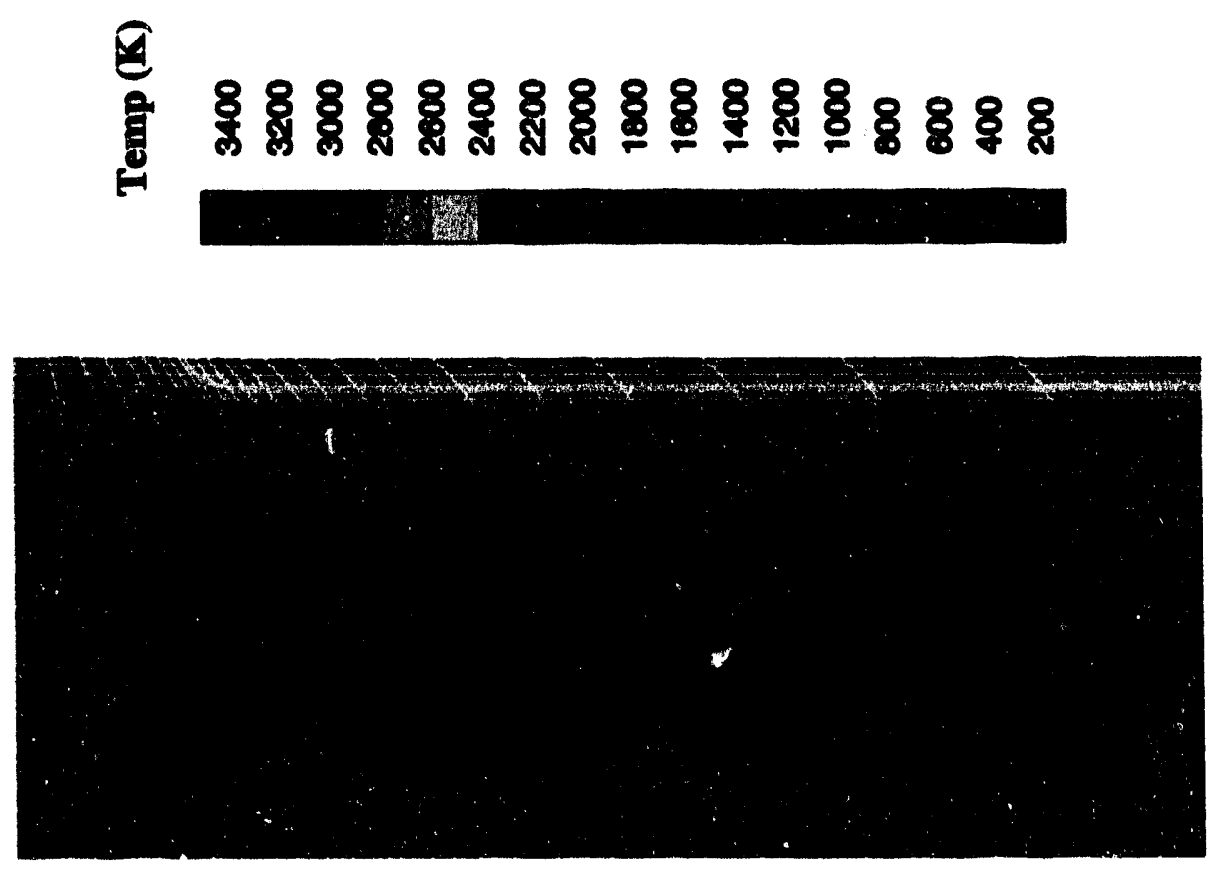

Figure 7: Temperature contours $1 \mathrm{sec}$ after transition. 

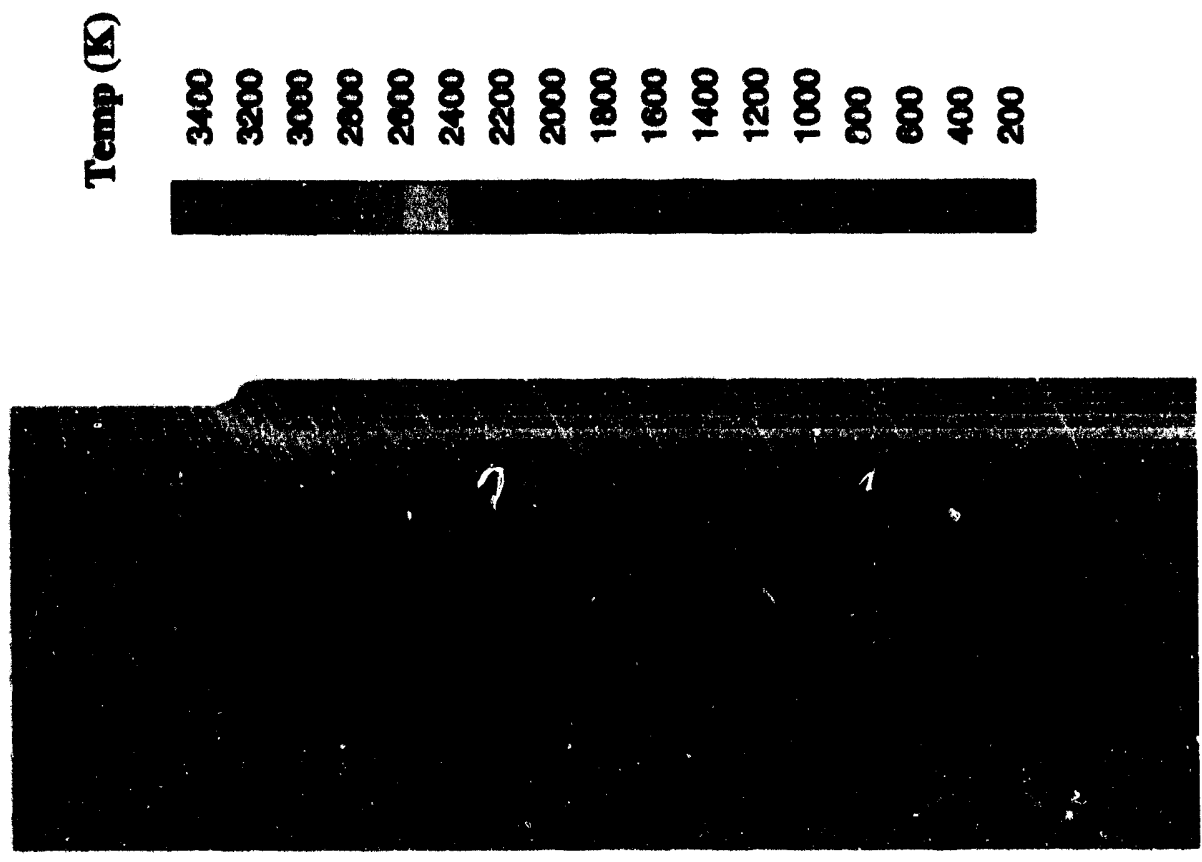

Figure 8: Temperature contours $5 \mathrm{sec}$ after transition.

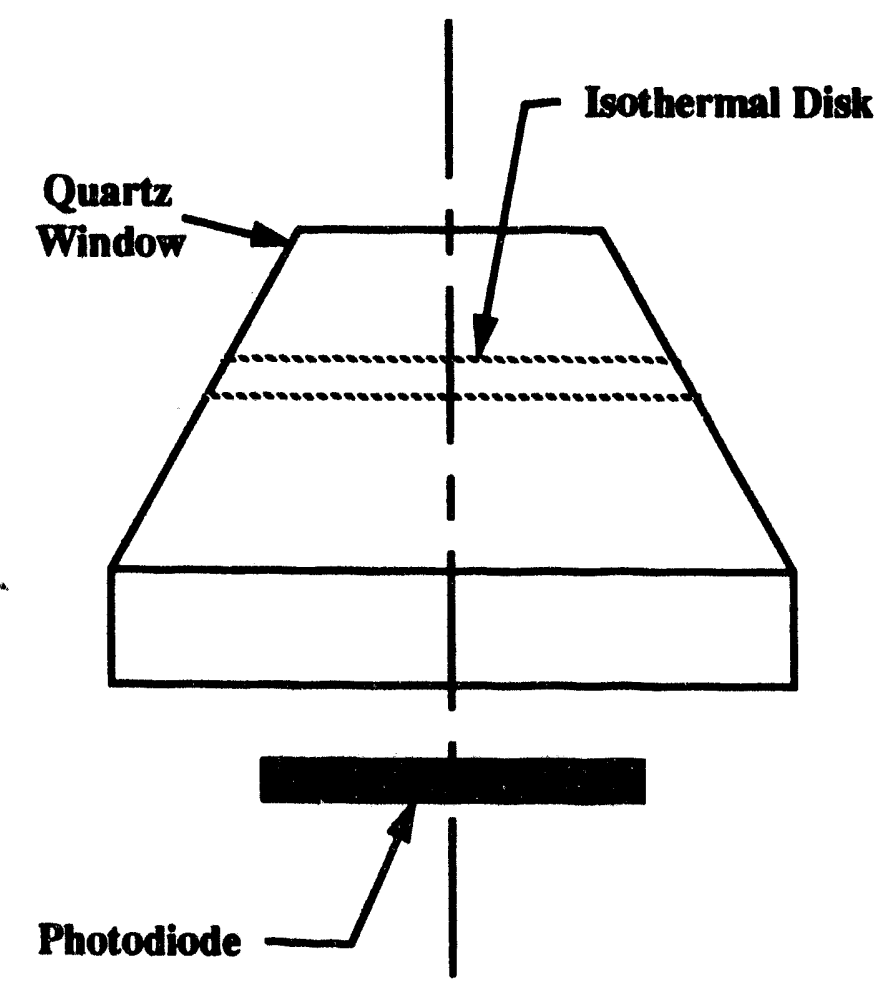

Figure 9: Radiating quartz model. 


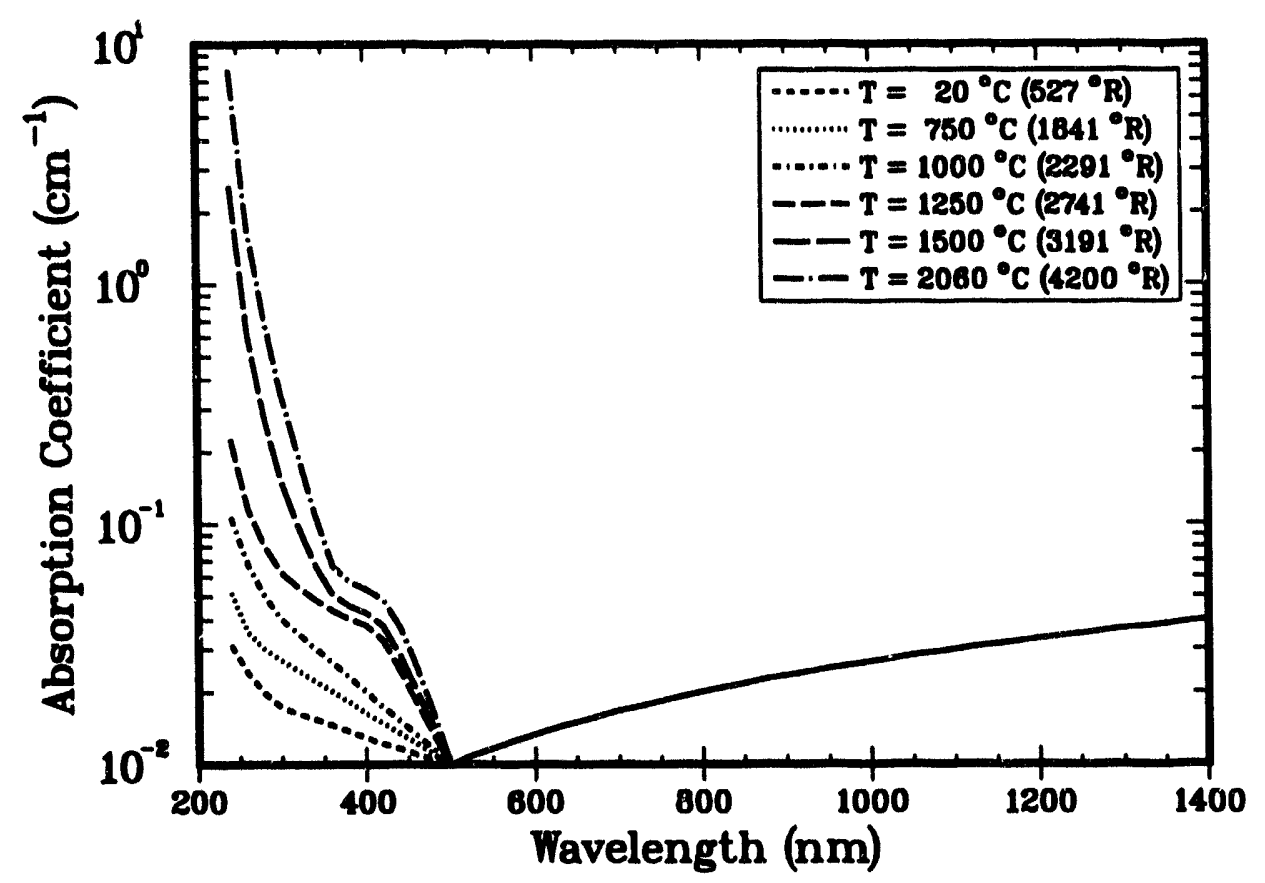

Figure 10: Quartz absorption coefficients obtained from Reference 9 $\left(2060^{\circ} \mathrm{C}\right.$ values are extrapolated).

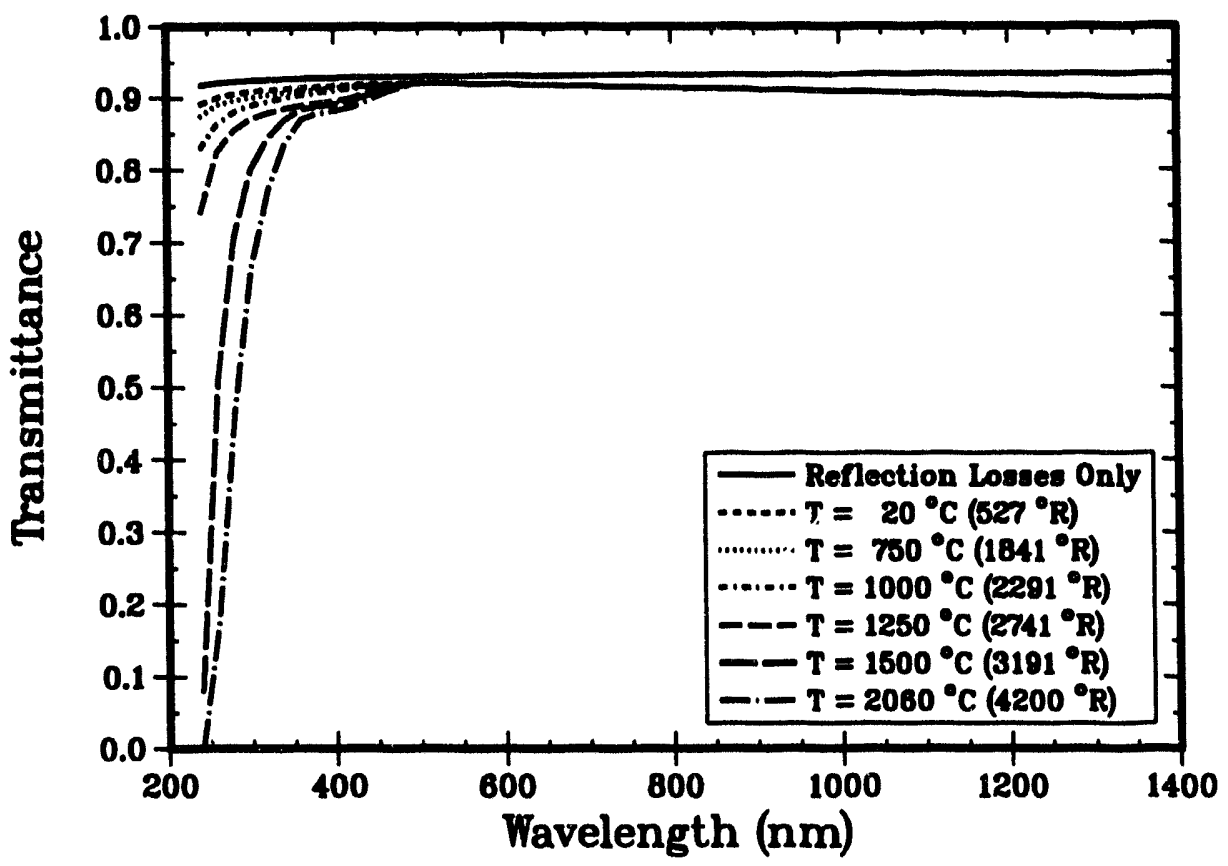

Figure 11: Quartz transmissivity obtained from Reference 9 ( $0.953 \mathrm{~cm}$ thick sample, $2060^{\circ} \mathrm{C}$ values are extrapolated). 


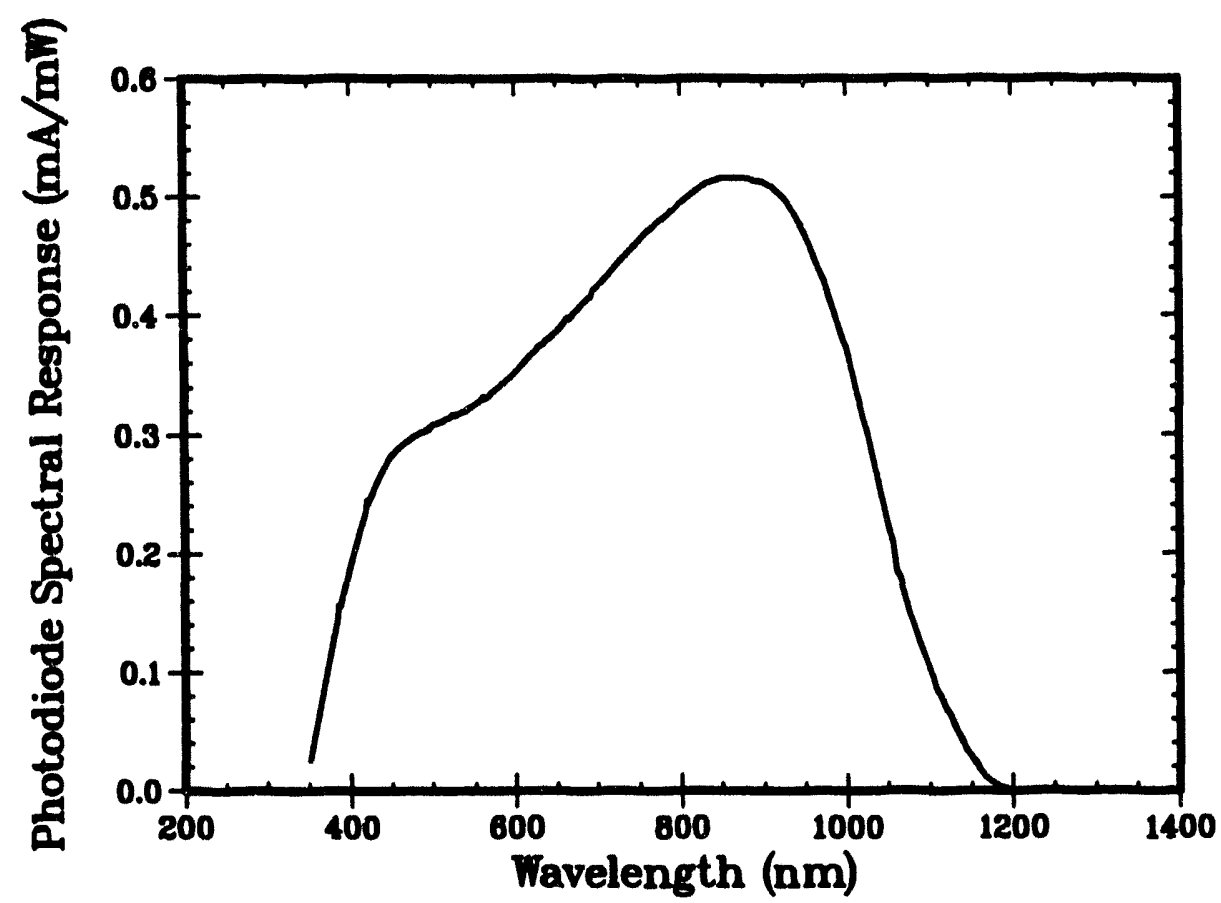

Figure 12: Photodiode spectral response.

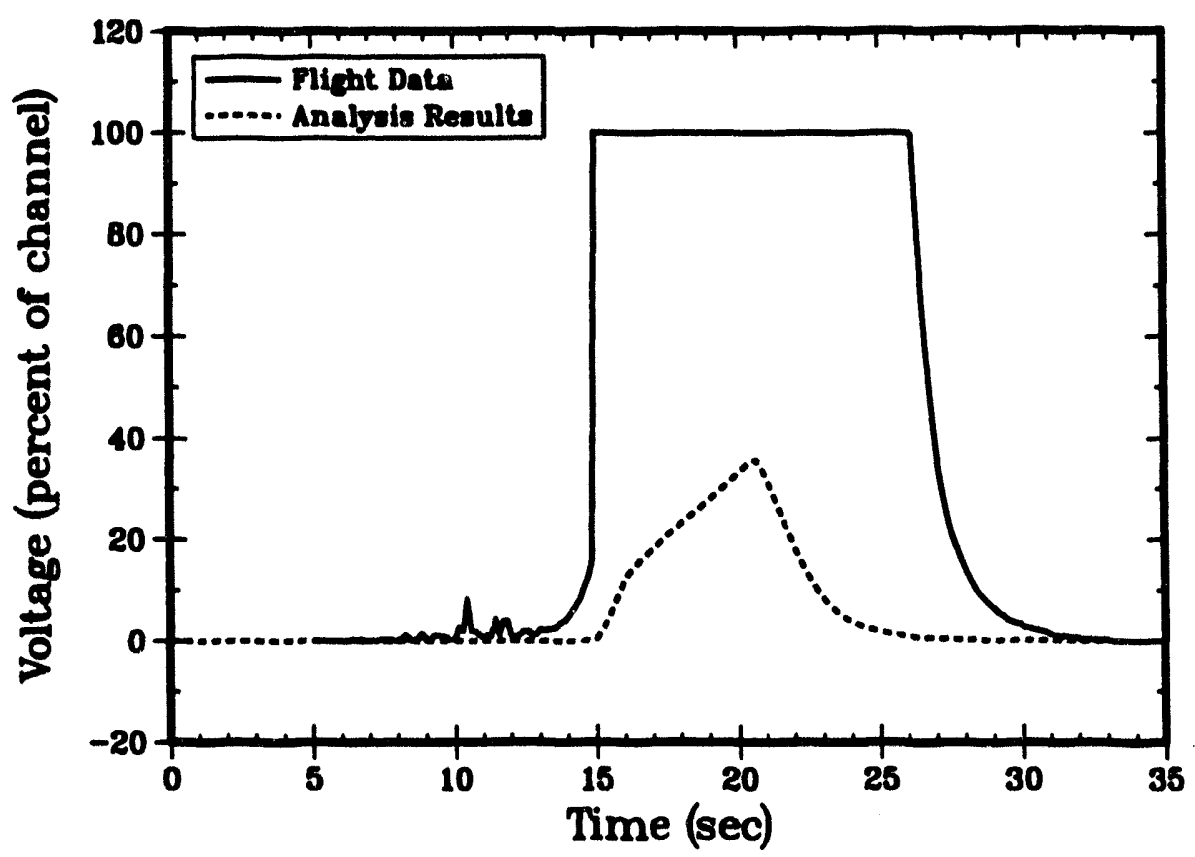

Figure 13: Radiating quartz analysis results for the photodiode indicating transition. 


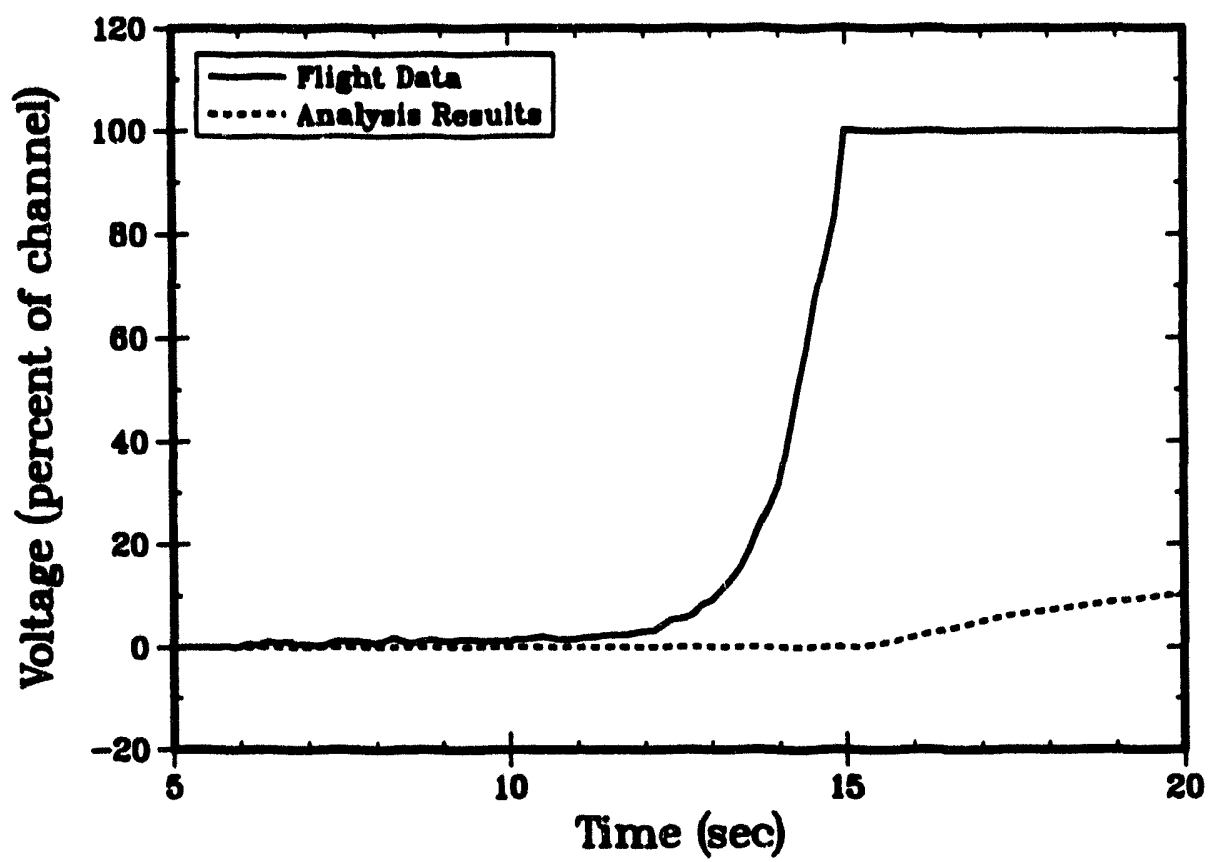

Figure 14: Radiating quartz analysis results for the photodiode saturating prior to transition. 


\section{Radiating Heatshield Material Analysis}

A second proposed source of the electromagnetic radiation detected by the photodiode involves the heatshield material surrounding the photodiode assembly. The relatively low thermal conductivity of the heatshield results in its surface temperature rising extremely rapidly at boundary layer transition to an ablation temperature of approximately $3,330 \mathrm{~K}$. Although the quartz window is surrounded by a thin layer of epoxy, the epoxy fails at a temperature of approximately $560 \mathrm{~K}$, and the heatshield material, at high temperatures, expands to fill any gap between the heatshield and quartz left by the failed epoxy. The relatively high emissivity of the heatshield material, and its high ablation temperature, indicate that adiation from the heatshield through the edges of the quartz window to the photodiode is a likely source of the sudden change in photodiode signal observed at boundary layer transition.

\subsection{Aerodynamic Heating Calculations}

The aerodynamic heating calculations used in this analysis are the same as those used in the radiating quartz analysis. Since cold-wall heating rates are calculated independently of material properties and surface temperatures, the same results apply to the quartz window material and the surrounding heatshield material.

\subsection{Material Thermal Response}

The two-dimensional material response computed with the SODDIT and FCV codes for the radiating quartz analysis was also used in the present analysis. The results of these computations provided the temperature history of the epoxy/heatshield material layer bordering the quartz window necessary for this analysis.

\subsection{Photodiode Response Model}

The photodiode response model for the radiating heatshield material analysis is similar to that used in the radiating quartz analysis. The conical/cylindrical window edge surface was divided into thin regions, as shown in Figure 15. These surfaces were assumed to be at a uniform temperature, equal to the temperature at the junction of the window and surrounding material at the depth of the region's center. Radiation from each of these surfaces was transmitted through the quartz window to the photodiode surface.

The current produced by the photodiode at each time step as a result of the radiation emitted by a single region of the surrounding heatshield can be determined from a relation similar to that used in the radiating quartz analysis:

$$
\mathrm{I}_{\mathrm{T}, \mathrm{hsr}}=\int\left(\mathrm{A}_{\mathrm{hsr}} \mathrm{F}_{\mathrm{hsr}-\mathrm{pd}} \in \mathrm{E}_{\mathrm{B}, \lambda} \tau_{\lambda, \mathrm{T}} \mathrm{R}_{\mathrm{pd}, \lambda}\right) \mathrm{d} \lambda
$$

In this equation, $A_{h s r}$ is the area of the isothermal conical/cylindrical heat shield region, $F_{h s r-p d}$ is the view factor from the heatshield region to the photodiode (approximated by a relation for conical surfaces radiating to disks, obtained from Reference 8 ), $\varepsilon$ is the emissivity of the heatshield and epoxy material (assumed to be a constant value of 0.85 ), $E_{B, \lambda}$ is the spectral emissive power of a blackbody radiator at the same temperature as the heatshield region, $\tau_{\lambda, T}$ is 
the total transmissivity of the quartz between the radiating surface and the photodiode, including a double surface reflective loss, and $R_{\mathrm{pd}, \lambda}$ is the spectral response curve of the photodiode (shown in Figure 12).

The total transmissivity of the quartz between the radiating surface and the photodiode, $\tau_{\lambda, T}$, was obtained from a similar relation as that used in the radiating quartz analysis:

$$
\tau_{\lambda_{r} T}=K \prod\left[\operatorname { e x p } \left(-\alpha_{\left.\left.\lambda_{r} T^{t_{q d}}\right)\right]}\right.\right.
$$

Here, the product is taken over all the quartz disks between the ra liating surface and the backface of the window. The quantity $\mathrm{K}$ is the maximum theoretical transmittance and is obtained from the relation:

$$
\mathbf{K}=(1-\mathbf{R})^{2}
$$

where $R$ is again the single surface reflection loss. This equation differs from that used in the radiating quartz analysis in that the quantity (1-R) is squared to account for both the reflective losses at the quartz surface next to the radiating surface and the reflective losses at the base of the window.

Similar to the radiating quartz analysis, the total current emitted by the photodiode at a given time due to radiation from the heatshield/epoxy surface is determined by summing the contributions from each conical/cylindrical region, thus including the effects of radiation from the complete window boundary. The load voltage is then determined, as in the radiating quartz analysis, by taking the product of the total current and the resistance.

\subsection{Radiating Heatshield Material Results}

The results of the radiating heatshield analysis are compared with flight data obtained from the center photodiode in Figure 16. The model predicted the nearly instantaneous rise in photodiode signal at boundary layer transition, and predicted reasonably well the signal rise prior to transition and the signal drop-off during the deceleration portion of the flight. Considering the accuracy with which aerodynamic heating and material response can be predicted, this agreement is considered quite good, indicating that radiation from the heatshield material surrounding the quartz window is very likely the primary source of the photodiode signal.

The results of the radiating heatshield analysis are compared with the forward photodiode flight data in Figure 17. Here, this analysis predicts that the photodiode which saturated prior to transition should have produced a good transition indication. Thus, it is apparent that the source of the signal which causes early photodiode saturation does not come from blackbody radiation from the heatshield material, and an additional source must be responsible. 


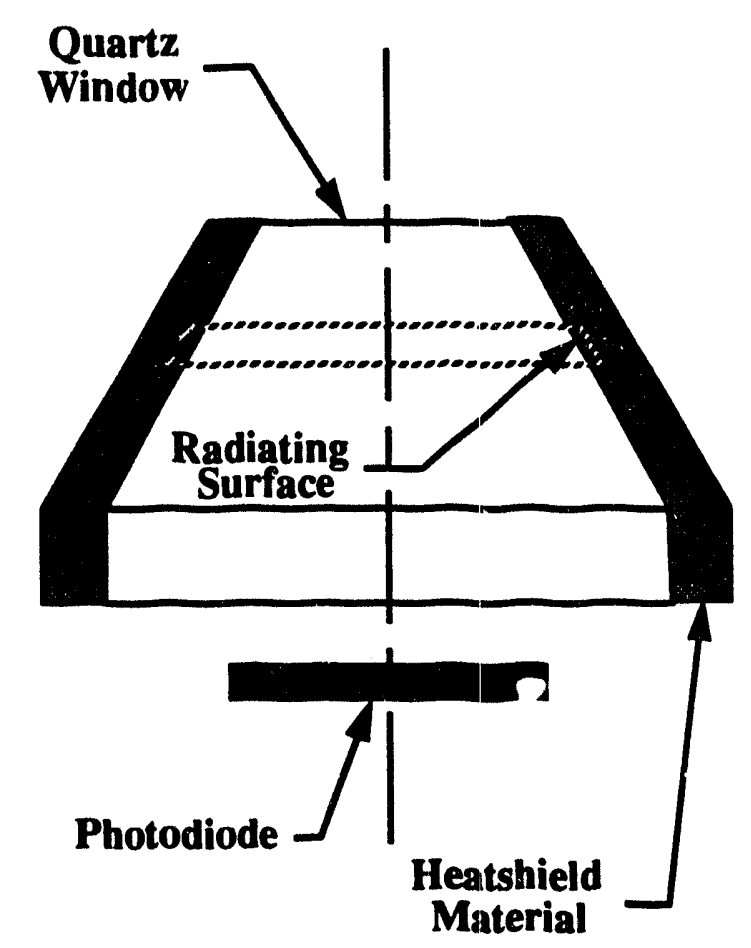

Figure 15: Radiating heatshield model.

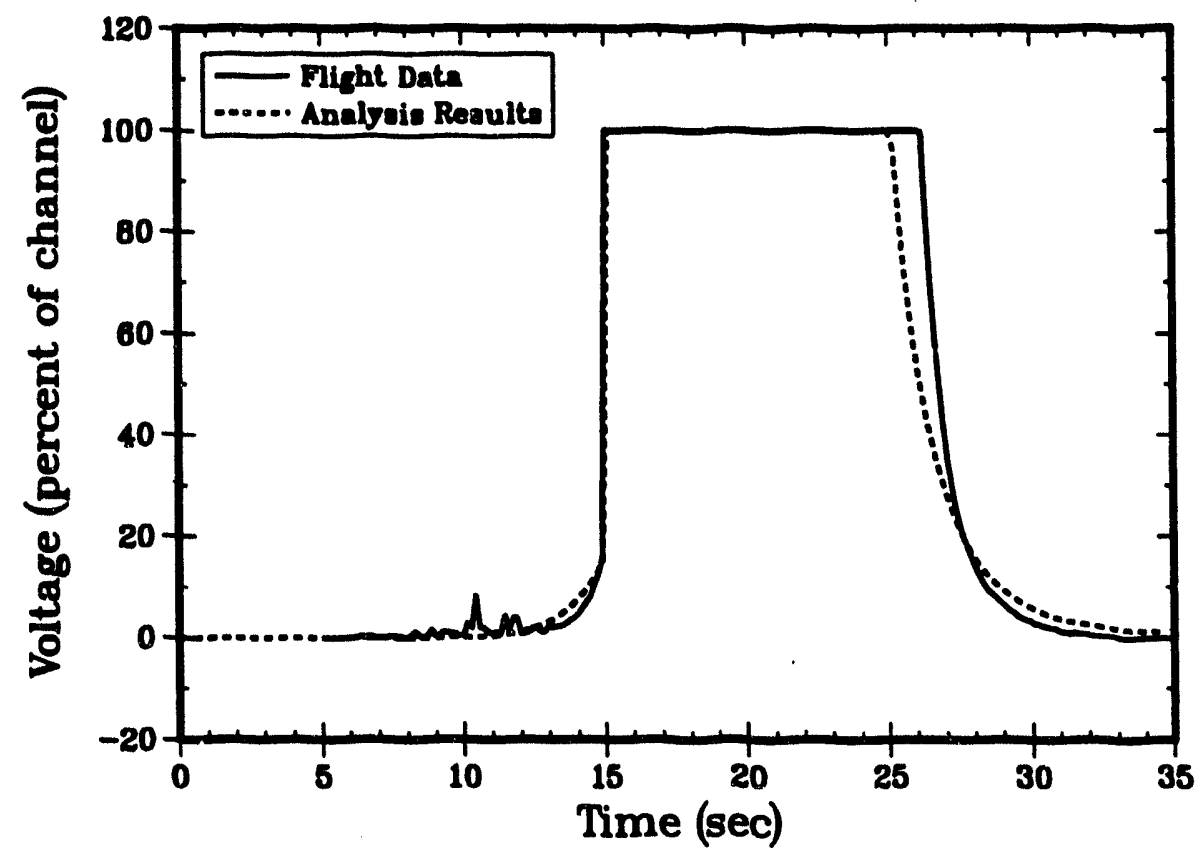

Figure 16: Radiating heatshield analysis results for the photodiode indicating transition. 


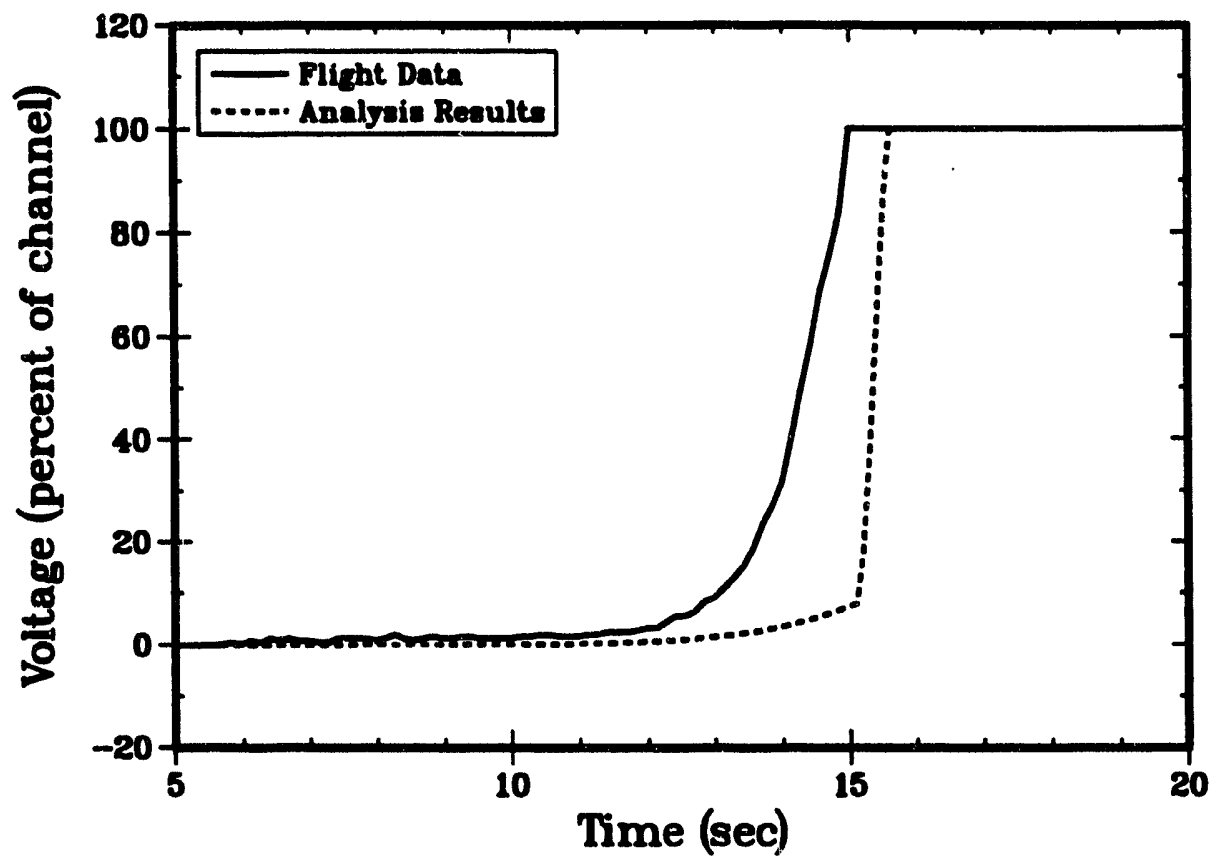

Figure 17: Radiating heatshield analysis results for the photodiode saturating prior to transition. 


\section{Radiating Boundary Layer Analysis}

A third proposed source of the photodiode signal is radiative emissions from the gases within the boundary and shock layers above the surface of the quartz window. In order to determine the strength of these emissions, and the likelihood that they are responsible for part or all of either the photodiode signal increase seen at boundary layer transition or the early saturation of photodiodes in some situations, analyses were performed for the center and forward photodiodes whose signals were initially presented in Figures 4 and 5 . The flowfield solution, including chemistry, was computed with the INCA code ${ }^{10}$. The radiation from the boundary layer was computed with the GENRAD code, which uses Park's NEQAIR ${ }^{11-13}$ subroutine. The response of the photodiode to the calculated spectrum was determined with a photodiode response model similar to those described above. Due to the computational expense of the INCA solution, only two solutions were obtained, a laminar solution and a turbulent solution, for conditions which existed at a reference time of 15 seconds. This time corresponds to the time of boundary layer transition for the center photodiode shown in Figure 4, and the time of saturation for the forward photodiode shown in Figure 5.

\subsection{Flowfield Solution}

The flowfield in the vicinity of the reentry vehicle was calculated with the INCA code. The INCA code solves the Navier-Stokes equations which account for multi-chemical species and energy modes with thermochemical nonequilibrium.

The reaction rates are defined by the law of mass action and, for this simulation, Park's $\mathrm{s}^{14}$ two temperature model provided the coefficients for the Arrhenius expressions. In this model, the vibrational, electron and electronic temperatures are assumed to be equal, and the translational and rotational temperatures are assumed to be equal. The vibrational energy source term is accounted for using the Landau-Teller formula ${ }^{15}$. The vibrational temperature influences the reaction rates by assuming the rate coefficients for dissociation are functions of the geometrical mean temperature between the translational-rotational temperature and the vibrational-electronelectronic temperature. The turbulent case was addressed using the Baldwin-Lomax model ${ }^{16}$.

-An LU-SGS ${ }^{17,18}$ (lower-upper symmetric Gauss-Seidel) implicit finite-volume method was used to solve the conservative form of the Navier-Stokes equations. The inviscid fluxes for the present predictions were evaluated using the Steger-Warming ${ }^{19}$ flux-vector-splitting method. The diffusion terms were evaluated using standard central differences. To reduce computational time and memory usage in the matrix inversion, the source Jacobian due to the chemical source terms was replaced with an approximate diagonal chemical source Jacobian ${ }^{20}$. The LU-SGS algorithm approximately solves this system using two sweeps of a point Gauss-Seidel relaxation.

The flowfield solutions around the axisymmetric hypersonic flight vehicle discussed above were obtained for turbulent and laminar flow. Both cases where run at the same freestream conditions. The flow domain was divided into three zones; the nosetip region (125 by 85 grid points), the forward photodiode region ( 92 by 85 grid points), and the center photodiode region (20 by 85 grid points). In order to ensure reasonable accuracy in predicting the temperature profile near the wall, 15 to 20 points were maintained in the thermal layer along the body.

The flight conditions of the hypersonic flight vehicle were in a region where slight ionization occurs. Since it was possible that a small amount of ions could be the source of the radiative emissions, the 11 species, 31 reaction Park model was used. The modeling of turbulent reacting 
flowfields is a difficult task and the physics models are not completely adequate for reacting gas mixtures in the Navier-Stokes code employed. There are some weaknesses in the modeling and the turbulent solution should be viewed accordingly.

Figure 18 shows the calculated temperatures for both the laminar and turbulent solutions at the location corresponding to the center photodiode. The vertical axis is $\mathrm{r}^{*}$, the distance from the vehicle surface nondimensionalized by the distance from the vehicle surface to the shock wave. A wall temperature of $1,712 \mathrm{~K}$, obtained from material response calculations, was used for both INCA solutions. The temperatures for the laminar and turbulent solutions are nearly identical for $r^{*}$ values greater than 0.6 , but differ significantly from this point to the vehicle surface where viscous effects would be expected to have the greatest effect. In the region from $r^{*}=0.1$ to $\mathrm{r}^{*}=0.6$, the turbulent case temperatures are both higher than their laminar counterparts. However, near the wall $\left(r^{*}<0.1\right)$, the turbulent translational-rotational temperature is greater than the laminar value, while the turbulent vibrational-electron-electronic temperature is less than the laminar value.

Figures 19 through 22 show the calculated molecular and ion mass fraction distributions for the laminar and turbulent cases. Significant dissociation of nitrogen and oxygen occurs only in the highest temperature regions of the boundary layer, with ion mass fractions being low in the entire flowfield. The differences in the calculated temperatures between the laminar and turbulent flowfields result in some differences in the calculated mass fractions. The most significant differences occur very near the wall, where the effects of the peak temperatures lying closer to the wall in the turbulent flowfield compared to the laminar flowfield can be seen.

\subsection{Boundary Layer Emissions}

The results obtained from the INCA solutions were used as inputs to the GENRAD code to determine the spectra of the line emissions from the high temperature gases within the boundary and shock layers. Initial calculations indicated that the gases could be treated as optically thin, and thus the spectra were calculated with bin widths of 5 angstroms. Figures 23 and 24 present the calculated spectra for the laminar and turbulent cases, respectively. It can be seen in these figures that the majority of the spectral intensity is in the ultraviolet range, with the intensity falling by approximately an order of magnitude from a wavelength of $200 \mathrm{~nm}$, the shortest wavelength calculated, to a wavelength of $350 \mathrm{~nm}$, the lower cut-off wavelength of the photodiode response curve (Figure 12). The laminar and turbulent spectra are very similar, with only subtle differences resulting from the differences in the calculated temperatures and chemical compositions for each solution.

\subsection{Photodiode Response Model}

The photodiode response model for the spectral gas emissions is similar to that used in the radiating quartz analysis. The product of the laminar or turbulent spectrum and the photodiode spectral response is integrated over wavelength, taking into account both the absorption of the quartz window as a function of wavelength and temperature, and the geometry of the photodiodewindow assembly, using the relation shown below:

$$
I_{T, q d}=\int\left(A_{w s} F_{w s-p d} 2 \pi S_{\lambda} \tau_{\lambda, T} R_{p d, \lambda}\right) d \lambda
$$


In this relation, $A_{w s}$ is the area of the window surface, $F_{w s-p d}$ is the view factor from the window surface to the photodiode (obtained from Reference 8 ), $S_{\lambda}$ is the spectral power obtained from the GENRAD code (originally in $W / \mathrm{cm}^{2}-\mu-s r$ ), $\tau_{\lambda, T}$ is the spectral transmissivity of the quartz window, including a double surface reflective loss, and $R_{p d, \lambda}$ is the spectral response curve of the photodiode. The $2 \pi$ is included to remove the steradiancy factor within the spectrum.

The results of these calculations indicate that the photodiode response to the radiation emitted from the gases within the boundary and shock layers is less than 0.1 percent of the photodiode saturation level for laminar and turbulent solutions for both photodiodes. This is a result of the low spectral emissions within the wavelength band detected by the photodiode. Thus, it is apparent that emissions from the high temperature gases cannot be responsible for either the signal change seen at boundary layer transition, as presented in Figure 4, or the early saturation of photodiode signals, as shown in Figure 5.

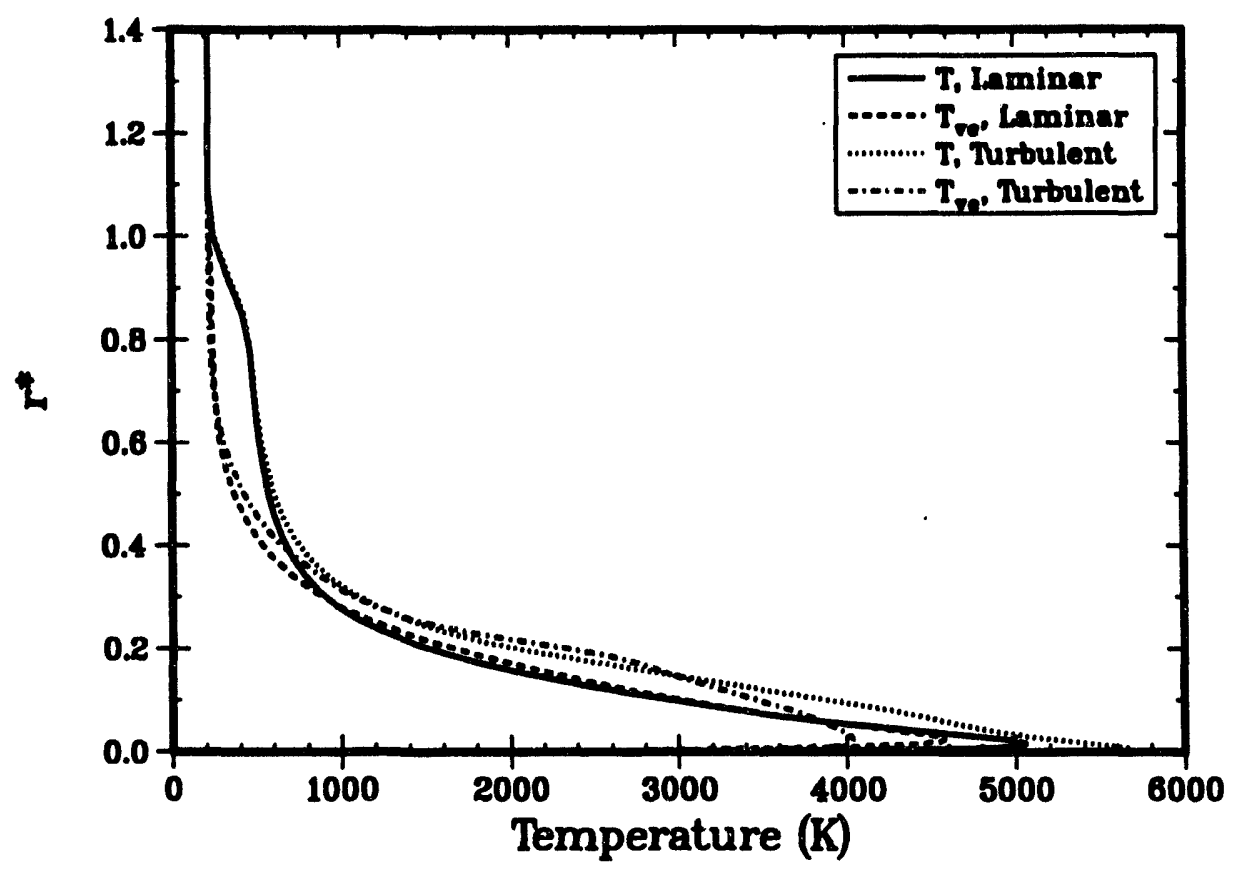

Figure 18: Calculated shock layer temperatures. 


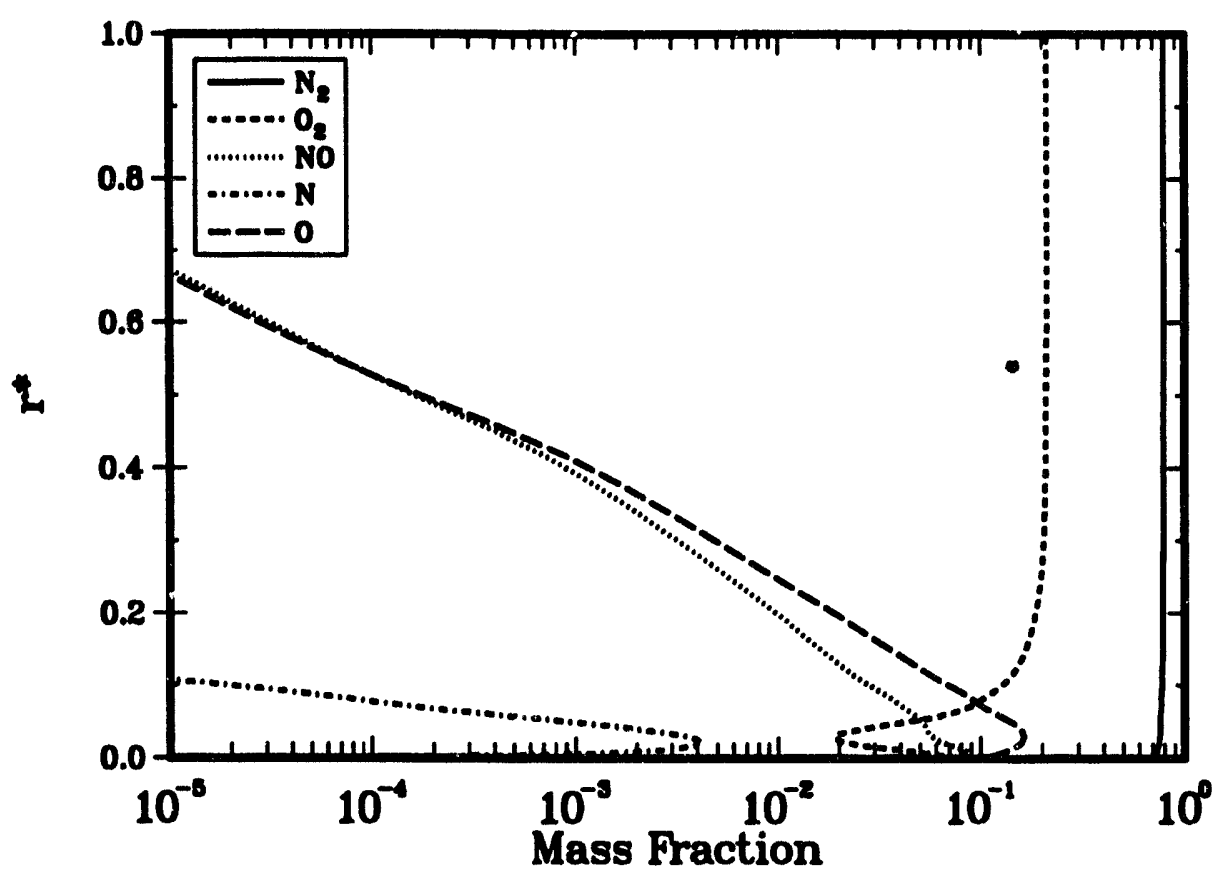

Figure 19: Calculated laminar mass fractions.

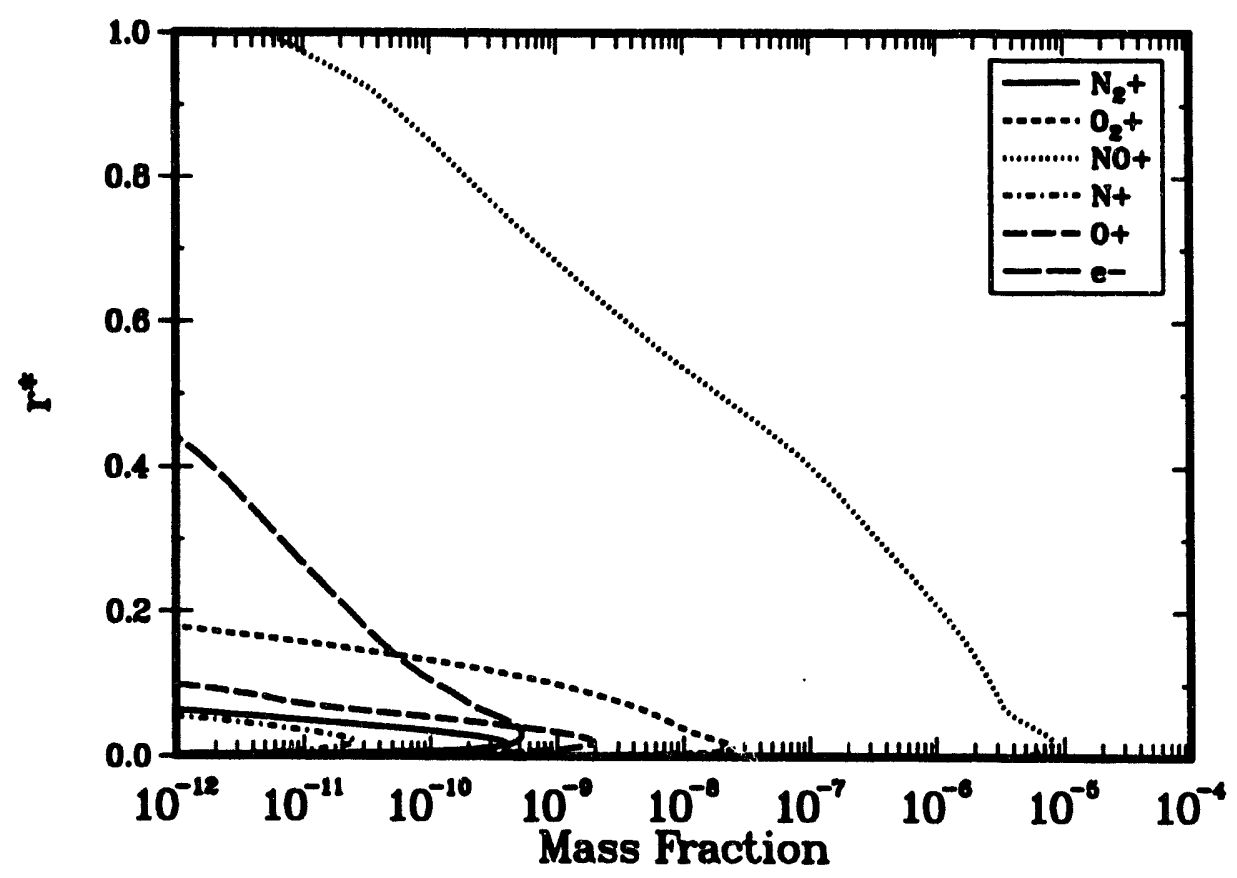

Figure 20: Calculated laminar ion mass fractions. 


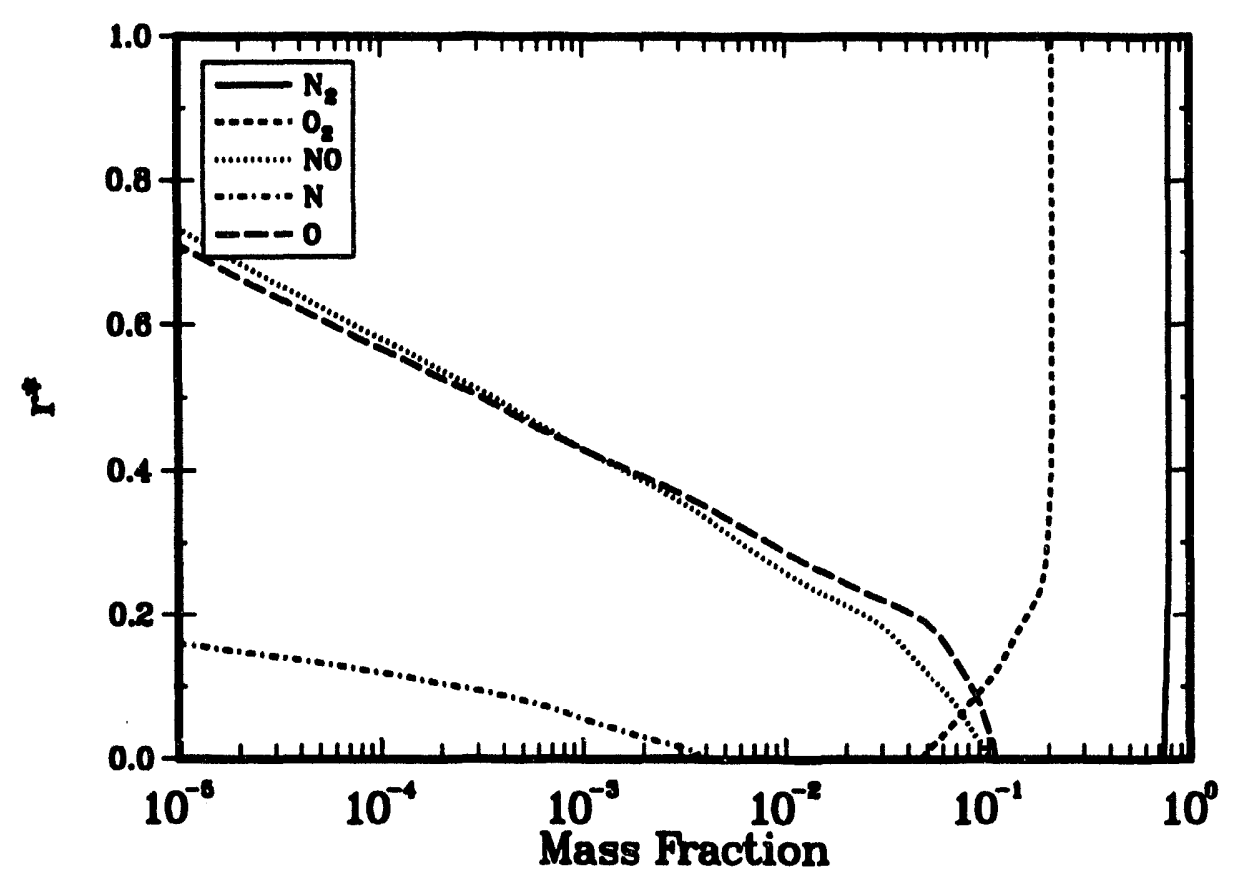

Figure 21: Calculated turbulent mass fractions.

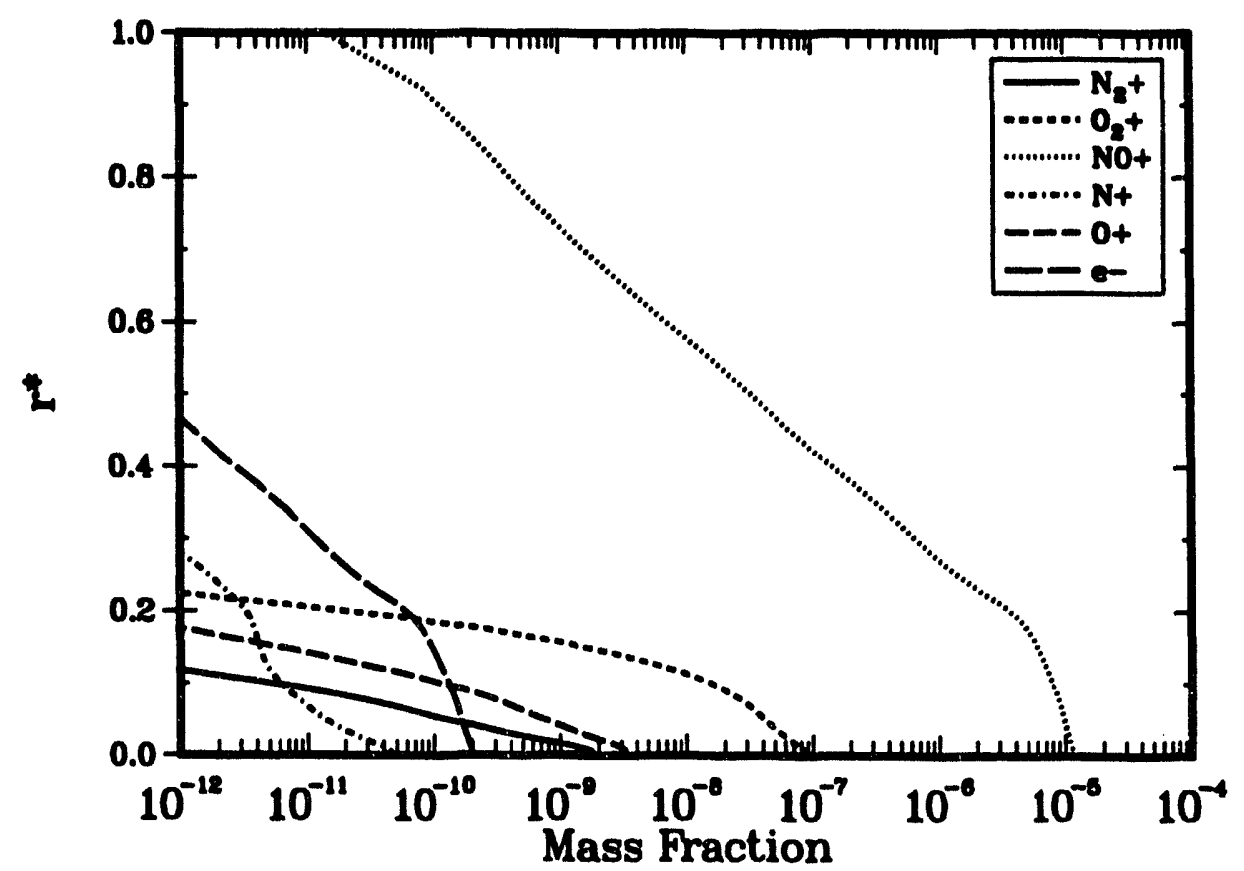

Figure 22: Calculated turbulent ion mass fractions. 


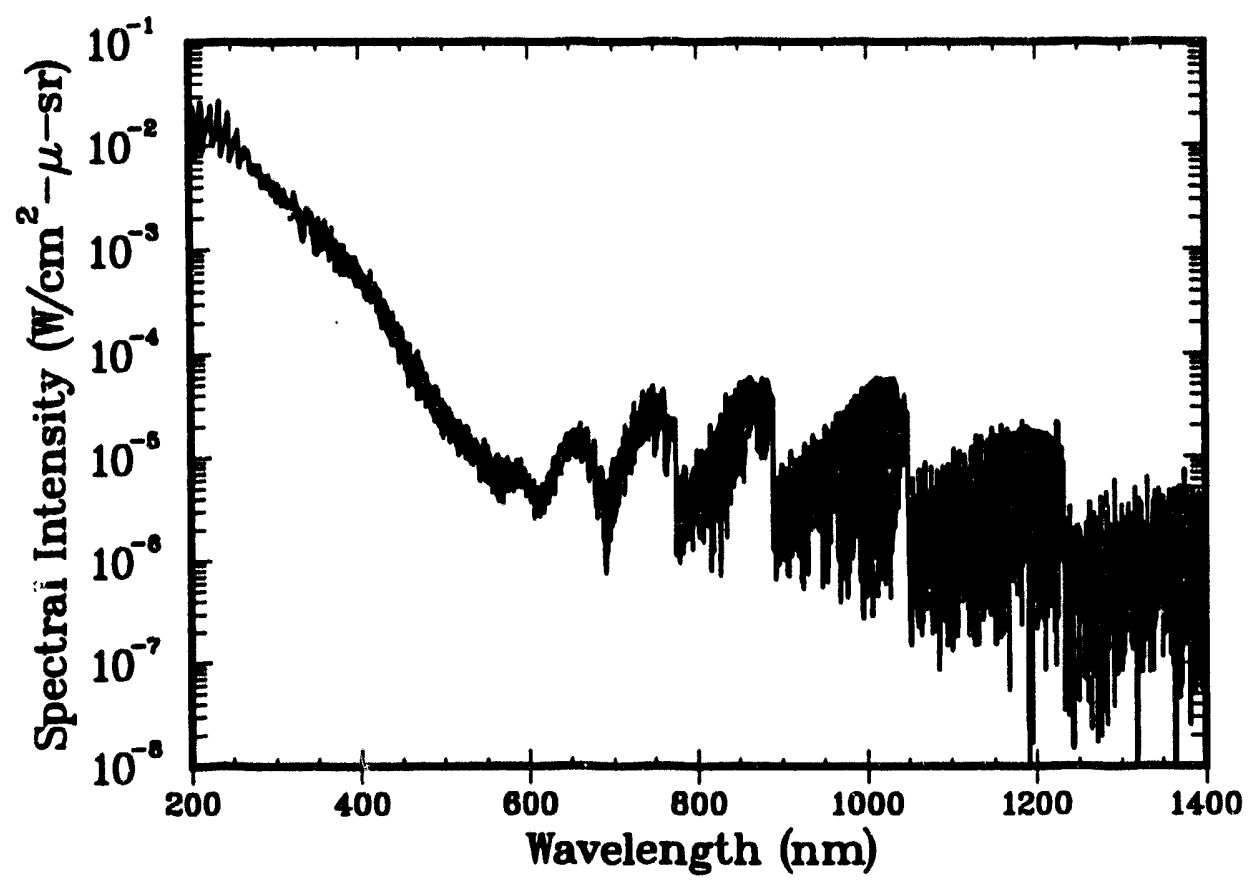

Figure 23: Calculated laminar spectrum.

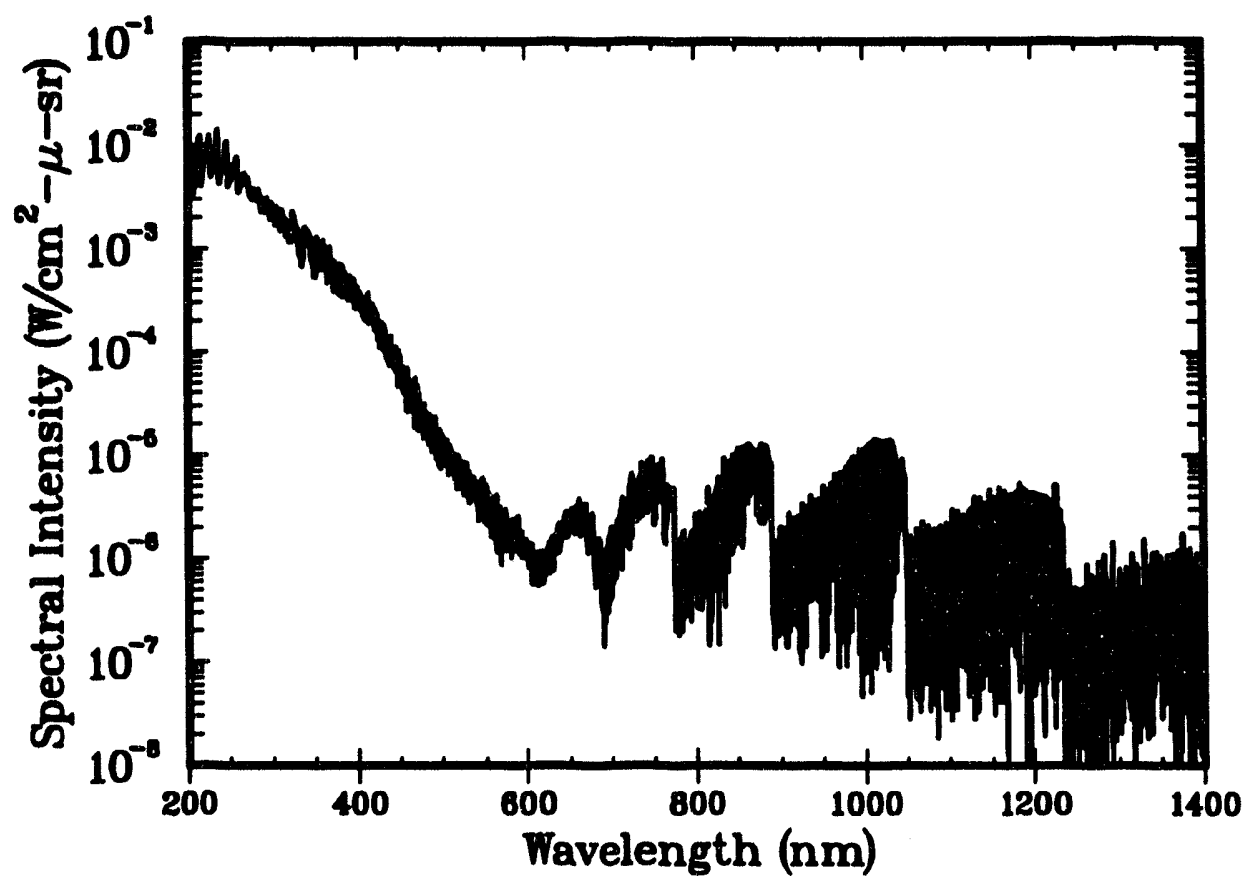

Figure 24: Calculated turbulent spectrum. 


\section{Radiating Ablation Products Analysis}

A fourth proposed source of the photodiode signal is radiative emissions from the ablation products produced by the nosetip and heatshield upstream of the photodiode window. These ablation products can be in the form of carbon compounds produced by chemical reactions between the carbon and the high temperature gases in the boundary layer, or in the form of solid carbon particles which have broken away from the nosetip or heatshield and are entrained within the gases near the surface of the vehicle. Chemical compounds resulting from the ablation of the phenolic within the heatshield were not considered in this analysis. Analyses were performed to investigate the possibility that radiation from ablation products results in an appreciable photodiode signal for the center and forward photodiodes whose signals were inifially presented in Figures 4 and 5. These analyses were performed for a reference time of 15 seconds, similar to the analysis which determined the emissions from the high temperature gases within the shock layer.

\subsection{Carbon Compound Concentrations}

The carbon ablation rate from the vehicle nosetip and heatshield prior to boundary layer transition was calculated from a combination of flight data and analysis techniques. The nosetip recession rate was measured during the flight and was used to estimate the carbon ablation rate from the spherical portion of the nosetip. The ablation rates on the conical portion of the nosetip and the heatshield were calculated using the Charring Materials Ablation code (CMA) ${ }^{21}$ with the heating rates obtained from the GE2IT' - SANDIAC ${ }^{3}$-HIBLARG ${ }^{4}$ family of codes assuming completely laminar flow. It should be noted that the CMA code removes material as a result of chemical reactions only, and does not model mechanical removal of solid carbon due to such phenomenon as cracking and spalling. These ablation rates were then integrated over the vehicle surface area upstream of the two photodiodes in order to determine the total carbon mass flow rate over the vehicle at the photodiode stations at the analysis time. The results of these analyses indicate that, at the reference time for a laminar boundary layer, approximately 62 percent of the carbon removal is occurring on the spherical portion of the nosetip, 38 percent is occurring on the conical portion of the nosetip, and an insignificant amount is occurring on the heatshield.

In order to determine the carbon concentration in the region above the photodiode windows, it was necessary to estimate both an average velocity of the ablation products over the surface of the vehicle, and the extent into the flowfield that the carbon products have penetrated due to diffusion processes. For this analysis, the penetration depth of the carbon into the flowfield was assumed to be equal to the laminar boundary layer thickness, which was calculated using the HIBLARG code. The mean velocity of the ablation products was set equal to the mass-averaged mean velocity within the boundary layer. Using these quantities, the mass fraction of the carbon in the laminar boundary layer in the vicinity of the center photodiode prior to transition was computed to be approximately 0.026 , and the mass fraction of the carbon in the laminar boundary layer in the vicinity of the forward photodiode prior to transition was computed to be 0.060 .

The carbon within the boundary layer at a photodiode station just after the transition front had passed over the window results from the laminar ablation processes occurring on the nosetip and heatshield forward of the transition front, and from the turbulent heatshield ablation occurring aft of the transition front and forward of the photodiode. To calculate the carbon concentration for this case, the GE2IT-SANDIAC-HIBLARG-CMA calculations were repeated with the boundary 
layer transitioning at a point approximately $15 \mathrm{~cm}$ upstream of the center photodiode station at a reference time of 14.8 seconds. The increase in heating rate associated with boundary layer transition resulted in a significant amount of carbon introduced into the boundary layer just forward of the photodiode. Using the turbulent boundary layer thickness at the photodiode location as the diffusion depth of the carbon, and the mass-averaged velocity within the turbulent boundary layer as the mean carbon velocity, the mass fraction of carbon within the turbulent boundary layer at the center photodiode location was computed to be approximately 0.018 . In spite of the increase in the amount of carbon being entrained within the boundary layer, this value is lower than that obtained for the laminar case due to the increase in boundary layer thickness resulting from transition, and the increase in the mass-averaged velocity within the boundary layer resulting from transition.

\subsection{Carbon Compound Spectral Emissions}

The equilibrium species concentrations present within the boundary layer for both the all laminar case and the turbulent case for the center photodiode were calculated with the Aerotherm Chemical Equilibrium ( $\mathrm{ACE})^{22}$ code using the carbon concentrations described above and the temperatures and pressures calculated with the INCA code. Equilibrium chemistry was used for this analysis both for simplicity, and due to the fact that, for the laminar case, the majority of the carbon within the boundary layer comes from the nosetip, which is a significant distance upstream of the photodiode locations, thus allowing a significant amount of time for the carbon-air chemistry to reach equilibrium prior to the ablation products reaching the photodiode locations. Figures 25 and 26 show the calculated carbon concentrations within the laminar and turbulent boundary layers, respectively, at the center photodiode location. The higher translational-rotational temperatures within the turbulent boundary layer result in significantly higher concentrations of carbon compounds and ions which extend further from the vehicle surface due to the increase in boundary layer thickness.

The carbon concentrations within the boundary layer, along with the air species concentrations computed by the ACE program within $t \mathrm{t} e$ boundary layer, were used as input for the GENRAD code. The air species concentrations from the INCA solutions were used in the region between the boundary layer edge and the shock wave. The spectra obtained from the GENRAD code for the laminar and turbulent solutions for the center photodiode are presented in Figures 27 and 28, respectively. The addition of carbon ablation products to the boundary layers makes a significant change to the spectra presented in Figures 23 and 24, with several groups of strong lines added near a wavelength of $400 \mathrm{~nm}$. The greater concentration of carbon compounds in the turbulent case results in somewhat higher spectral intensities. However, in both cases, the mean spectral curve drops off above a wavelength of $400 \mathrm{~nm}$, similar to what was observed in the spectra without carbon compounds present.

The photodiode response model for the carbon-air spectral emissions is identical to that used in the high temperature air spectral analysis. The product of the spectral laminar or turbulent emission and the photodiode spectral response is integrated over wavelength, taking into account both the absorption of the quartz window as a function of wavelength and temperature and the geometry of the photodiode-window assembly. The results of these calculations, similar to the results of the air spectral analysis, indicate that the photodiode response to the radiation emitted from the combination of the ablation products and gases within the boundary and shock layers is very low, less than 0.2 percent of the photodiode saturation level for the center photodiode 
laminar case and less than 0.5 percent for the center photodiode turbulent case. This again is a result of the relatively low spectral emissions within the wavelength band visible to the photodiode. Thus, it is apparent that emissions from the high temperature ablation products and gases cannot be responsible for either the signal change seen at boundary layer transition as presented for the center photodiode in Figure 4 , or the early saturation of photodiode signals, such as that obtained from the forward photodiode and shown in Figure 5.

The relative strengths of the radiation reaching the center photodiode surface are illustrated in Figure 29. The vertical axis of the graph is the photodiode spectral power in Watts/micron. This quantity is the optical power, as a function of wavelength, which reaches the surface of the photodiode, taking into account the geometry of the device and the absorption and reflection losses within the quartz window. Curves are presented for the radiation from the quartz window material, radiation from the heatshield material bordering the window, radiation from the air within the boundary and shock layers, and radiation from the carbon-air compounds within the boundary and shock layers. All four curves are for turbulent flow at a time of $15 \mathrm{sec}$. These curves illustrate that at short wavelengths, less than approximately $450 \mathrm{~nm}$, the radiation incident on the photodiode is primarily due to spectral line emissions from the carbon and air components within the boundary and shock layers. At wavelengths greater than approximately $450 \mathrm{~nm}$, the radiation striking the photodiode is primarily the result of blackbody radiation from the heatshield material bordering the quartz window.

Figure 30 presents the photodiode spectral current, in Amps/micron as a function of wavelength. This quantity is the product of the photodiode spectral power, shown in Figure 29, and the photodiode spectral response, shown in Figure 12. These curves are integrated over wavelength to obtain the total photodiode current. This figure further illustrates that above a wavelength of approximately $450 \mathrm{~nm}$, the photodiode signal is dominated by radiation from the heatshield material, which is approximately two orders of magnitude greater than the signals produced by the quartz window material and the carbon-air ablation products.

\subsection{Carbon Particle Blackbody Emissions}

The carbon particle analysis was performed primarily to determine if solid particles breaking away from the nosetip could result in the saturation of the forward photodiode, as shown in Figure 5, prior to boundary layer transition. While carbon particles breaking away from the heatshield just aft of the transition front could contribute to the photodiode signal change resulting from transition, no analysis techniques exist for evaluating this possibility. The relatively low pressures on the heatshield surface, compared to the stagnation region of the nosetip, should result in very little mechanical carbon removal. However, this point cannot be thoroughly addressed with the analysis techniques available.

A significant amount of uncertainty also exists regarding the mechanical removal of carbon from the vehicle nosetip. This uncertainty includes such factors as the relative amount of carbon which is removed as particles, the size distribution of these particles, and the temperatures of these particles. Due to the lack of information concerning the ablation of the nosetip in the actual flight environment, a parametric analysis in particle size was performed to determine the possible effects of carbon particles on photodiode signals.

An initial analysis was performed to determine if particles removed by ablation processes from the nosetip could survive the boundary layer environment long enough to reach the photodiode 
locations and to determine approximate surface temperatures for these particles. For this analysis, 1,10 , and 100 micron diameter particles were removed from a location on the nosetip just aft of the sphere-cone tangency point. These particles were given an initial temperature equal to the calculated surface temperature at that point on the nosetip. A one-dimensional trajectory program was used to calculate the particle velocity and position over the vehicle as a function of time, using boundary layer edge conditions obtained from the HIBLARG code as the local flow conditions. The drag coefficients for these trajectory calculations were obtained from Reference 23 and are based on ballistic range data. The trajectory information was then used with the $\mathrm{HANDI}^{24}$ program to determine particle heating rate information. HANDI is a correlationbased code which solves numerous types of aero-heating related problems. The heating rate information was in turn used with the CMA code to determine particle temperature and ablation rate histories.

For each size particle used in this analysis, the calculated surface recession was small, with the largest relative surface recession calculated to be $11 \%$ of the radius for the 10 micron diameter particle. Figure 31 presents the calculated particle surface temperatures as a function of location on the vehicle. In each case, the particles undergo a rapid rise in temperature due to the initially high heating rates resulting from the large difference in velocity between the particles and the boundary layer gases. After the particles have accelerated to velocities relatively close to the boundary layer gases, the particles begin to cool, with the smaller particles cooling the most rapidly.

The photodiode signal resulting from carbon particles removed from the nosetip of the vehicle was computed in a similar fashion as the other signal sources described above. The calculations were performed parametrically in both particle size and temperature, assuming that all the mass removed from the nosetip was in particle form. The distribution of carbon within the boundary layer was sufficiently sparse such that the radiation from one particle toward the photodiode will not be blocked by other particles, and thus the radiation reaching the photodiode can be considered to be the superposition of the radiation from numerous particles. The particles were assumed to be spherical in shape, and the appropriate shape factor for the radiation calculation was obtained from Reference 8. Absorption by the quartz window as a function of wavelength was accounted for in the calculation, which involved integrating the product of the blackbody radiation reaching the photodiode and the photodiode response curve over wavelength. Figure 32 presents the results of these analyses for the center photodiode.

The results of this analysis indicate that if the particles are sufficiently small, on the order of 1 micron in diameter, and have a sufficient temperature, approximately $4000 \mathrm{~K}$, they could result in the saturation of a photodiode signal. These results, combined with the calculated particle temperature histories shown in Figure 31, offer a possible explanation for the early saturation of the forward photodiode while the center photodiode (and other photodiodes on the same vehicle) remained unsaturated until transition. Relatively small particles of carbon, removed from the nosetip due to thermal cracking and spalling, are initially aerodynamically heated to high temperatures as they accelerate within the boundary layer. The particles then cool as they move down the vehicle, causing a significantly higher signal in the photodiodes located on the forward portions of the vehicle than those located on the aft portions of the vehicle. The uncertainties, in the nosetip performance and its response to the heating environment prevent a more detailed analysis of this phenomenon, but this process does explain the difference in photodiode signals on 
this vehicle, as well as on a similar reentry vehicle which also had forward-mounted photodiodes saturate early while the aft-mounted photodiodes remained unsaturated until local boundary layer transition occurred.

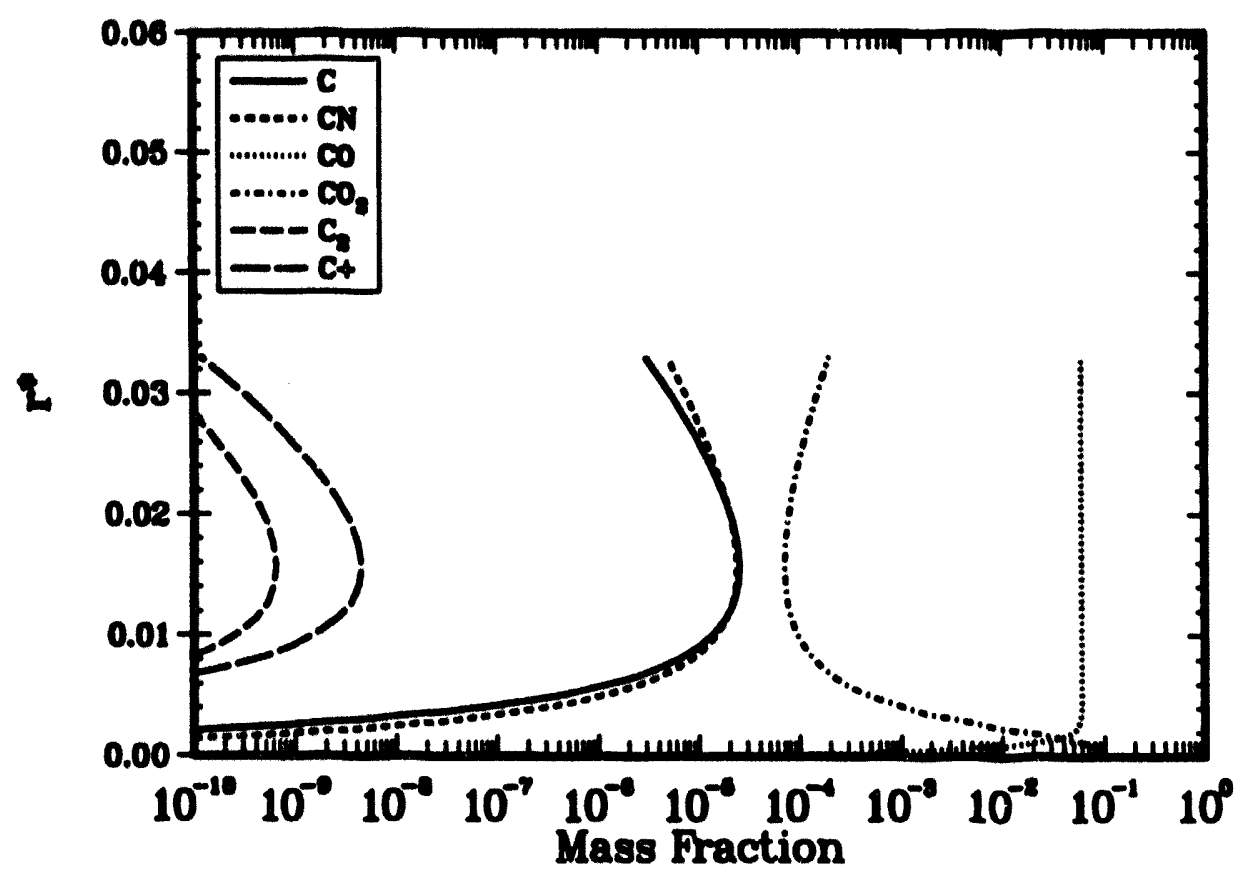

Figure 25: Calculated laminar carbon mass fractions. 


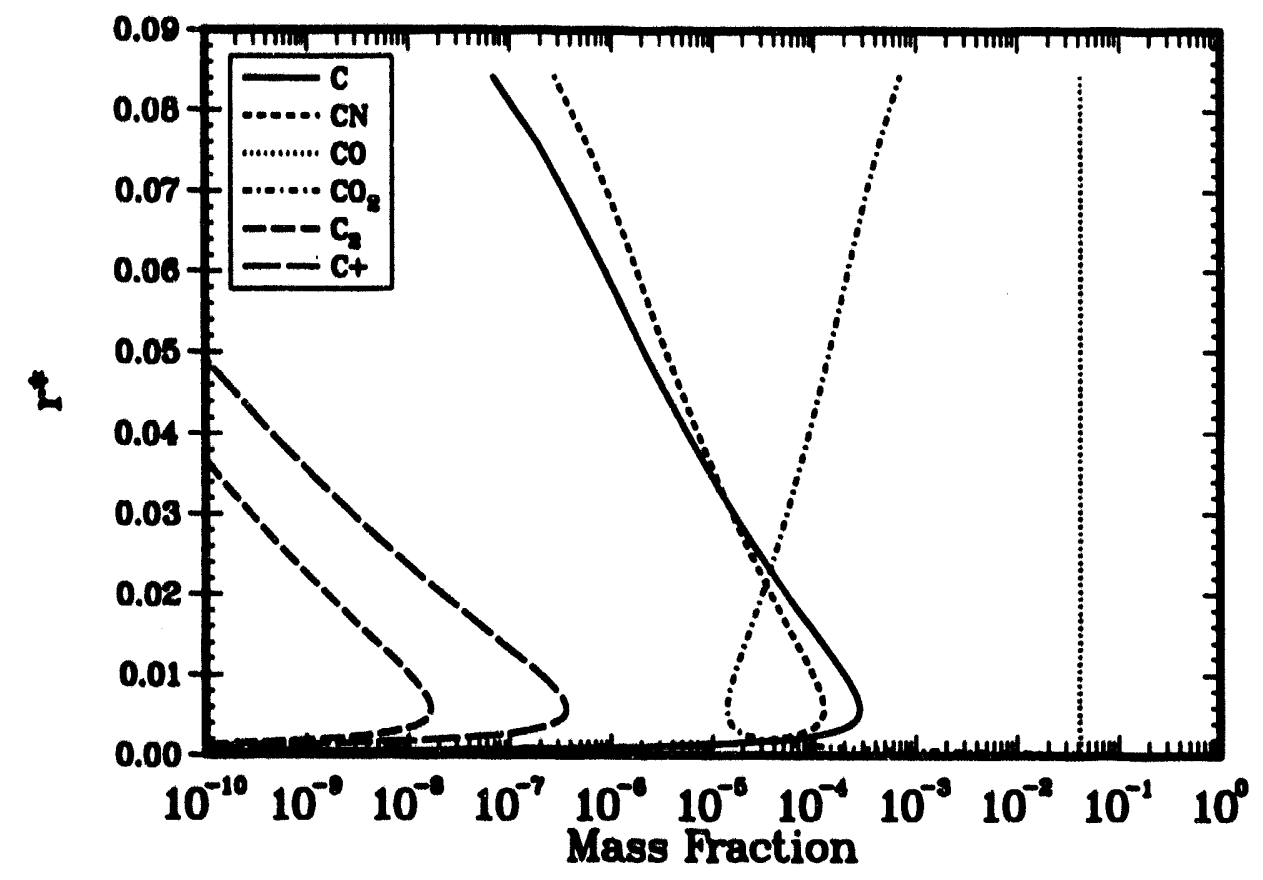

Figure 26: Calculated turbulent carbon mass fractions.

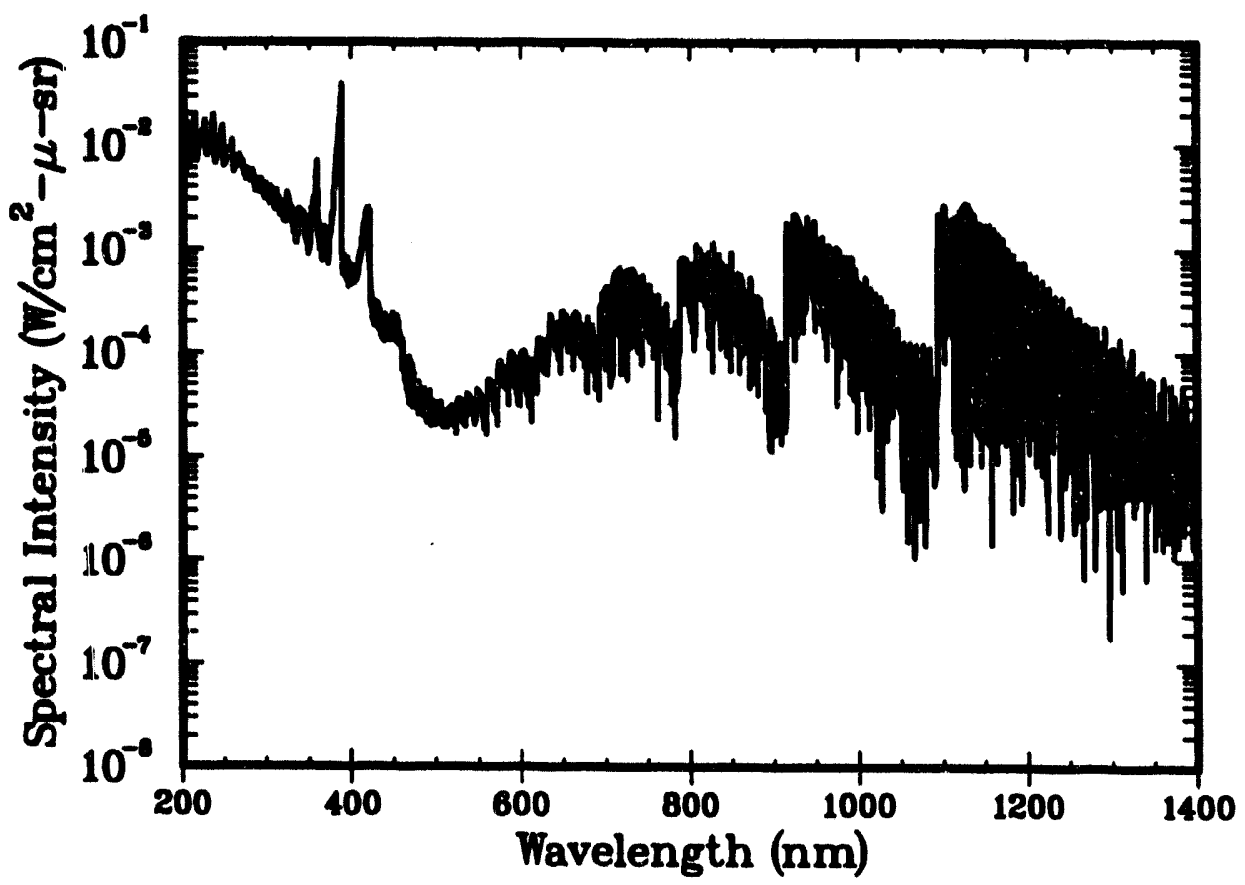

Figure 27: Calculated laminar carbon and air spectrum. 


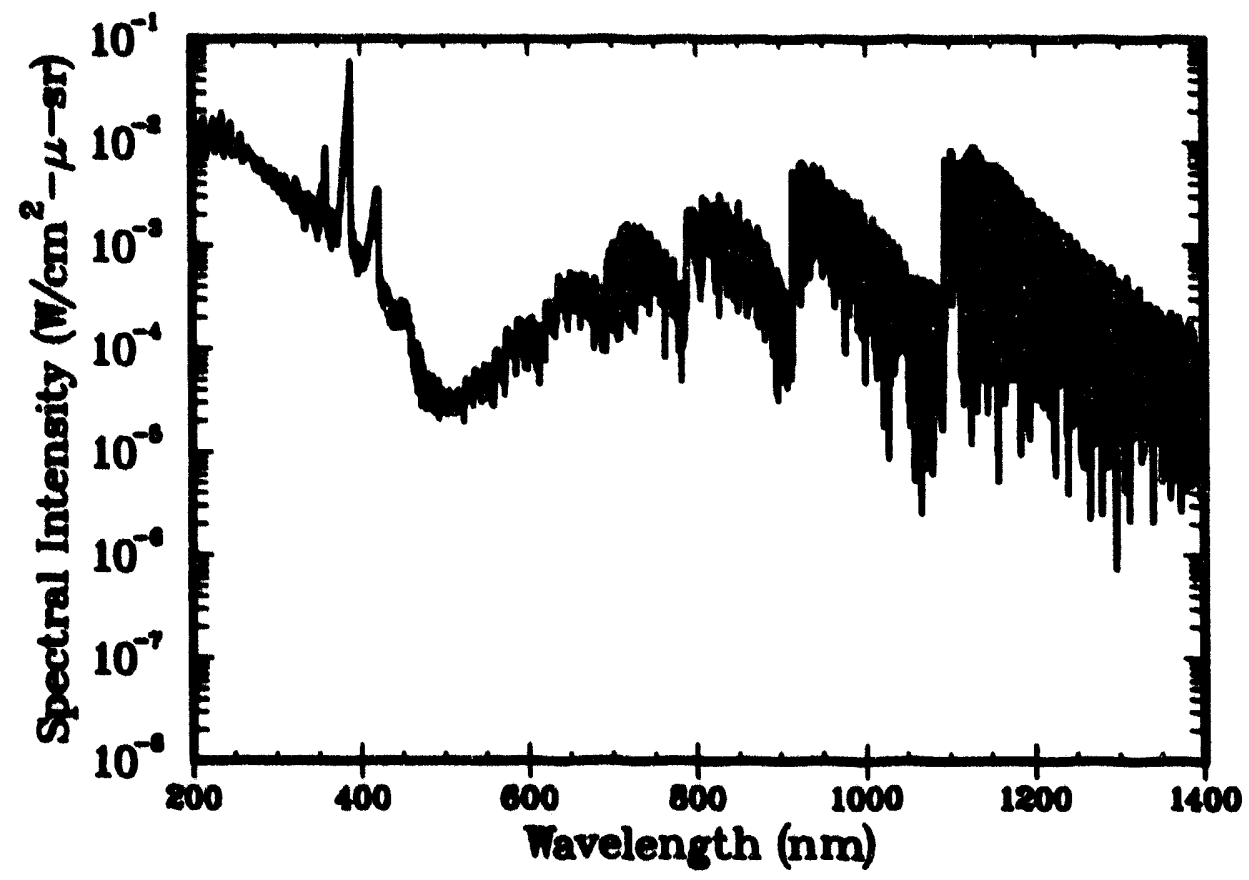

Figure 28: Calculated turbulent carbon and air spectrum.

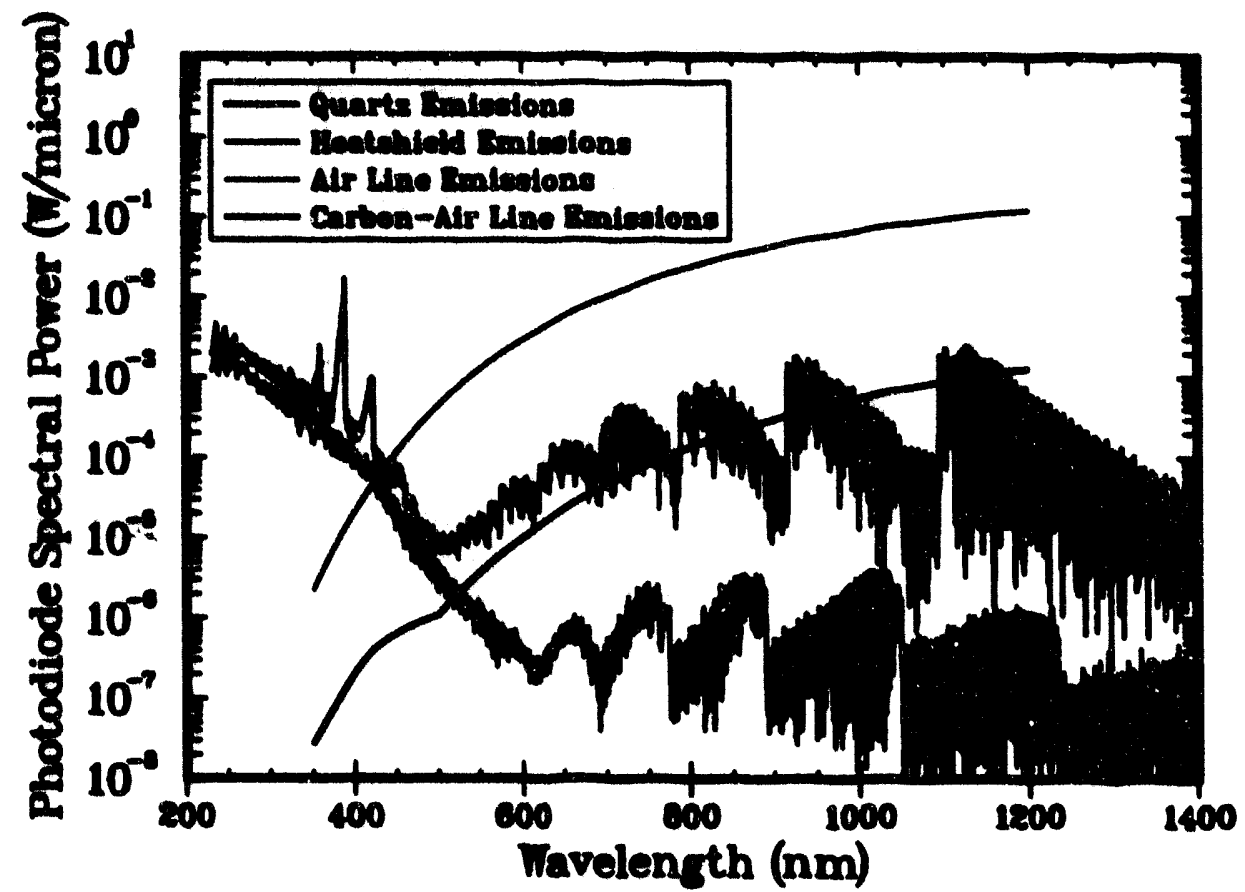

Figure 29: Comparison of photodiode spectral power. 


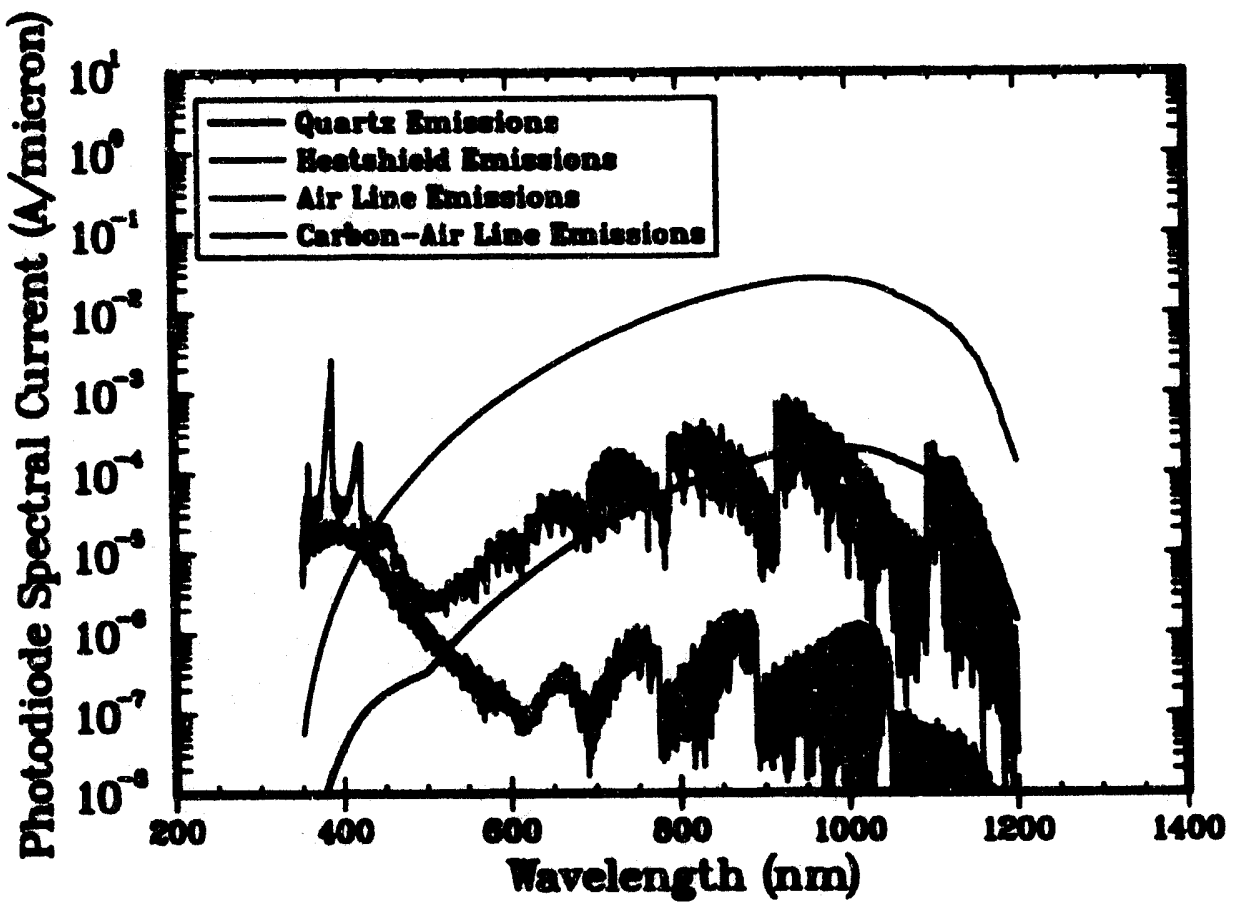

Figure 30: Comparison of photodiode spectral current.

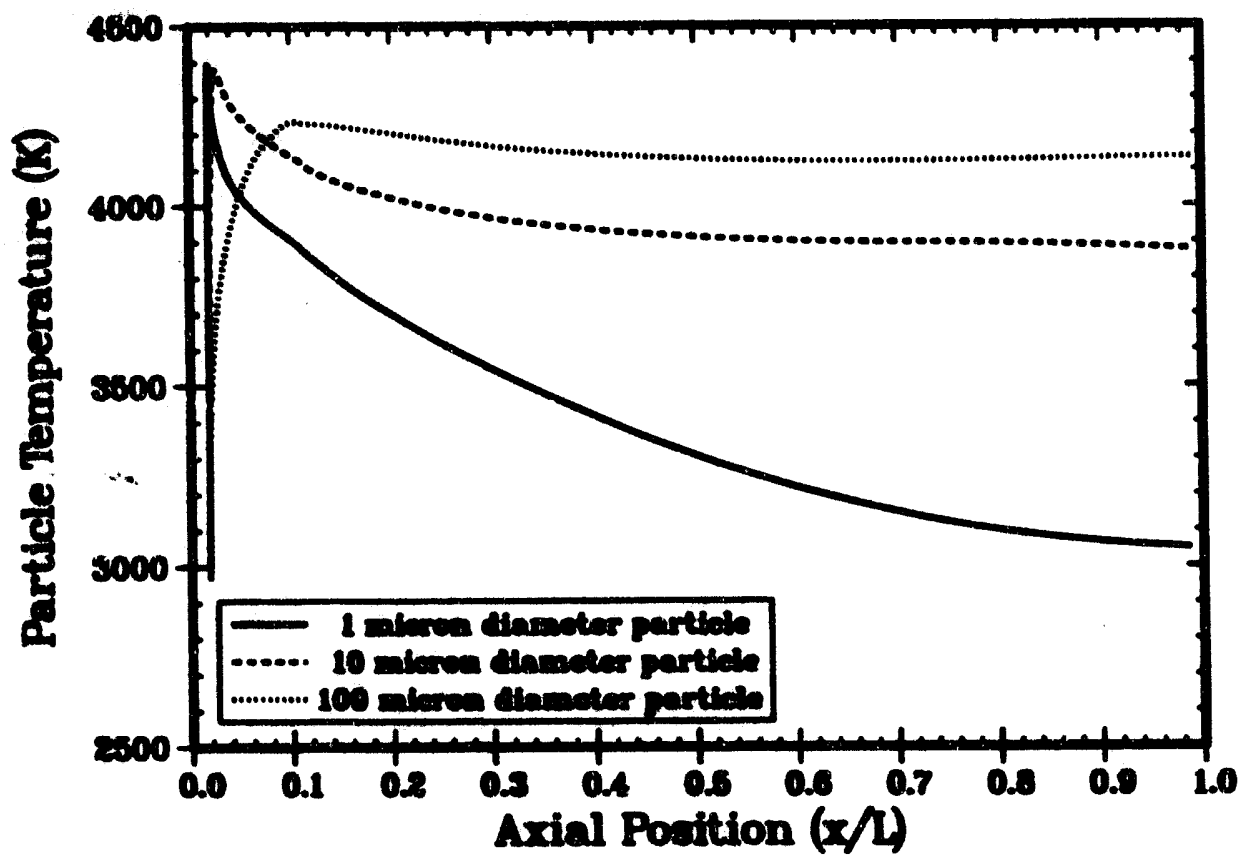

Figure 31: Calculated particle temperatures. 


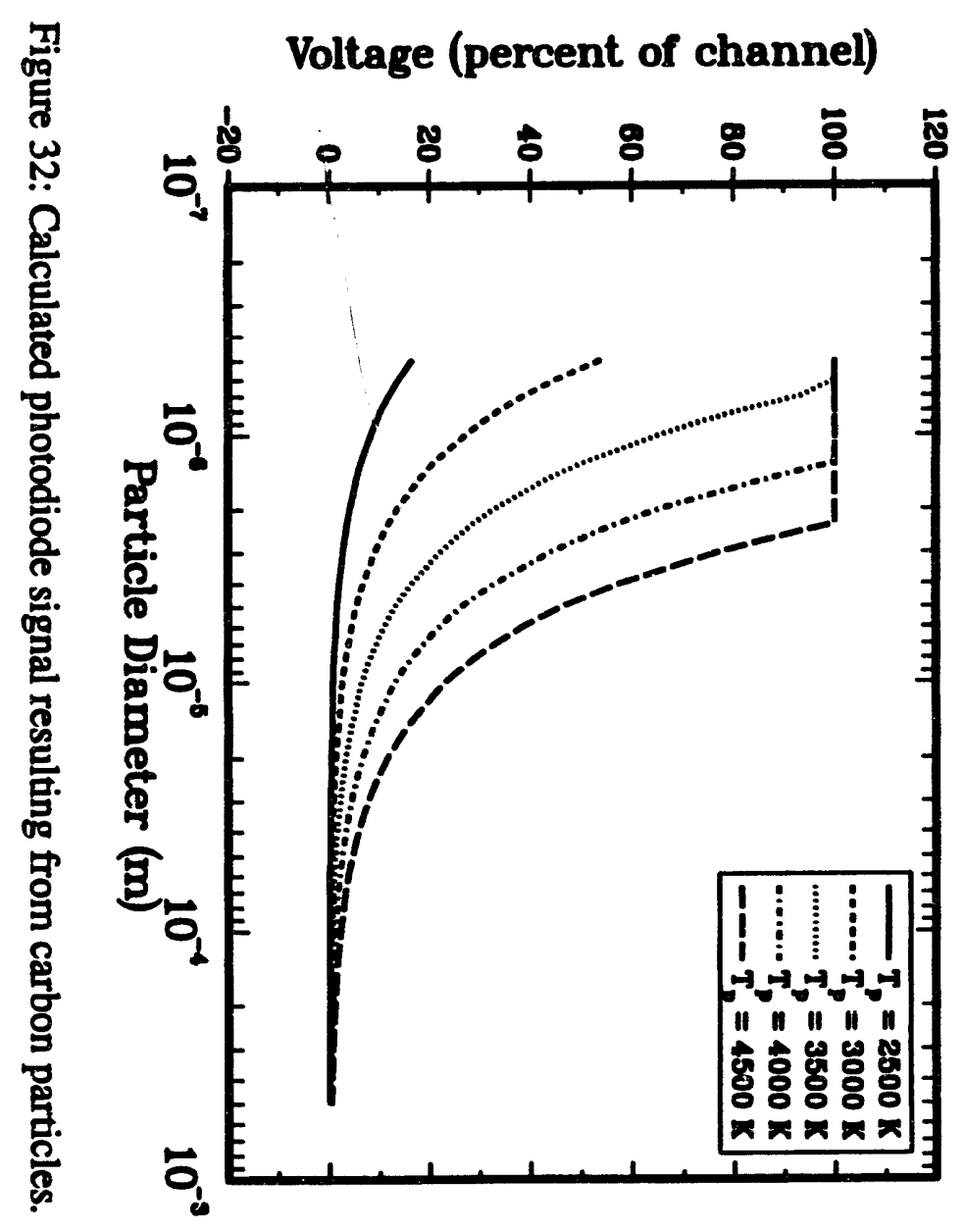


Page intentionally left blank. 


\section{Summary}

Analyses of the four proposed sources of the photodiode signal have been completed. The results of these analyses indicate that the most likely source of the radiation seen at boundary layer transition is emission from the heatshield material surrounding the quartz window. The originally proposed source of the radiation at boundary layer transition, emission from the quartz window material, is not sufficient to produce the signal observed in flight tests. In addition, spectral radiation from both the gases within the shock layer resulting from air chemistry and carbon ablation products is insufficient to be either the cause of the photodiode signal at transition or the early satu tion prior to transition. Although there is insufficient information available on the response of the nosetip material to the heating environment to perform a detailed analysis, parametric studies indicate that a likely source of the radiation which causes early photodiode saturation is blackbody radiation from carbon particles. It is proposed that these particles break away from the nosetip, are initially heated to relatively high temperatures, and then cool as they pass down the length of the vehicle. This theory explains the tendency for forward photodiodes to saturate while photodiodes further aft on the vehicle perform normally. 
Page intentionally left blank. 


\section{Acknowledgments}

This work was performed at Sandia National Laboratories which is operated for the DOE under contract number DE-AC04-94AL85000. The authors wish to thank Bennie F. Blackwell and Donald L. Potter, both of Sandia National Laboratories, for their assistance with the FCV and ACE codes, respectively, and Louis A. Gritzo and Vincent A. Amatucci, also of Sandia National Laboratories, for their assistance in the preparation of this manuscript. The authors also wish to thank Ellis Whiting of NASA-Ames Research Center for his valuable assistance with the GENRAD code. 
1 


\section{References}

[1] Blodgett, S. R., Conrad, B. T., Shrock, K. W., Wilken, A. C., Sterk, M. W., and Chaffin, R. J., "A Novel Reentry Vehicle Instrument - The Photodiode," ISA Transactions, Vol. 26, No. 3, pp. 19-23, July 1987.

[2] Daywitt, J., Brant, D., and Bosworth, F., "Computational Technique for Three-Dimensional Inviscid Flow Fields About Reentry Vehicles," General Electric Company, Philadelphia, Pennsylvania, April 1978.

[3] Noack, R. W., and Lopez, A. R., "Inviscid Flow Field Analysis of Complex Reentry Vehicles: Volume I, Description of Numerical Methods, Volume II, User's Manual," SAND87-0776, Sandia National Laboratories, Albuquerque, New Mexico, OctoberNovember 1988.

[4] Polansky, G. F., "Hypersonic Integral Boundary Layer Analysis of Reentry Geometries (HIBLARG) Code Description and User's Manual Version 2.0," SAND89-0552, Sandia National Laboratories, Albuquerque, New Mexico, March 1990.

[5] Thornton, A. L, "LOVEL-84: A Low-Velocity Aerodynamic Heating Code for Flat Plates, Wedges, and Cones," SAND84-0457, Sandia National Laboratories, Albuquerque, New Mexico, May 1984.

[6] Blackwell, B. F., and Hogan, R. E., "Numerical Solution of 2-D Axisymmetric Heat Conduction Problems Using a Finite Control Volume Technique," AlAA 91-1353, presented at the AIAA 26th Thermophysics Conference, Honolulu, Hawaii, June 24-26, 1991 (accepted for publication in the AIAA Journal of Thermophysics and Heat Transfer).

[7] Blackwell, B. F., Douglass, R. W., and Wolf, H., “A User's Manual for the Sandia OneDimensional Direct and Inverse Thermal (SODDIT) Code," SAND85-2478, Sandia National Laboratories, Albuquerque, New Mexico, May 1987.

[8] Howell, J. R., "A Catalog of Radiation Configuration Factors," to be published by McGrawHill Book Company.

[9] Beder, E. C., Bass, C. D., and Shacklefore, W. L., "Transmissivity and Absorption of Fused Quartz Between $0.22 \mu$ and $3.5 \mu$ from Room Temperature to $1500^{\circ} \mathrm{C}$," Applied Optics, Vol. 10, No. 10, October 1971, pp. 2263-2268.

[10] INCA: 3D Multi-Zone Navier-Stokes Flow Analysis with Finite-Rate Chemistry, User's Manual Part 1: Input Guide, Amtec Engineering, Inc., Bellevue, Washington, January 1992.

[11] Whiting, E. E., Arnold, J. O., and Lyle, G. C., "A Computer Program for a Line-By-Line Calculation of Spectra from Diatomic Molecules and Atoms Assuming a Voigt Line Profile," NASA TN D-5088, Ames Research Center, Moffett Field, California, March 1969.

[12] Park, C., "Nonequilibrium Air Radiation (NEQAIR) Program: User's Manual," NASA Technical Memorandum 86707, Ames Research Center, Moffett Field, California, July 1985.

[13] Moreau, S., Laux, C. O., Chapman, D. R., and MacCormack, R. W., "A More Accurate Non-Equilibrium Air Radiation Code: NEQAIR Second Generation," AIAA 92-2968, presented at the AIAA 23rd Plasma Dynamics and Lasers Conference, Nashville, Tennessee, July 6-8, 1992. 
[14] Park, C., "Assessment of Two-Temperature Kinetic Model for Ionizing Air," AIAA Journal of Thermophysics and Heat Transfer, Vol. 3, No. 3, pp. 233-244, July 1989.

[15] Vincenti, W. G., and Kruger, C. H., Introduction to Physical Gas Dynamics, Robert E. Krieger Publishing Company, Malabar, Florida, 1965.

[16] Baldwin, B. E., and Lomax, H., "Thin-Layer Approximation and Algebraic Model for Separated Turbulent Flows," AIAA 78-257, January 1978.

[17] Yoon, S., and Jameson, A., "An LU-SSOR Scheme for the Euler and Navier-Stokes Equations," AIAA 89-0600, 1987.

[18] Yoon, S., and Kwak, D., "Artificial Dissipation Models for Hypersonic External Flow," ALAA 88-3708, 1988.

[19] Steger, J. L., and Warming, R. F., "Flux Vector Splitting of the Inviscid Gasdynamic Equations with Applications to Finite-Difference Methods," Journal of Comp. Phys., Vol. 40, pp. 263-293, 1981.

[20] Eberhardt, S., and Imlay, S., "A Diagonal Implicit Scheme for Computing Flows with Finite-Rate Chemistry," AIAA-90-1577, 1990.

[21] Lauger, L. M., Kaestner, P. C., and Blackwell, B. F., "Operation Instructions for Charring Material Ablation Code," Sandia National Laboratories Report No. SLA-73-0745, Sandia National Laboraturies, Albuquerque, New Mexico, 1973.

[22] Powars, C. S., and Kendall, R. M., "Aerotherm Chemical Equilibrium (ACE) Computer Program," Aerotherm Corporation, Mountainview, California, May 1969.

[23] Kuntz, D. W., and Amatucci, V. A., "Drag and Heating Modifications Implemented Within the PLUTO Computer Program," SAND92-2519, Sandia National Laboratories, Albuquerque, New Mexico, January 1993.

[24] Potter, D. L., Memo to W. L. Hermina, Subject: HANDI Capability update \& Sun Conversion, Sandia National Laboratories, Albuquerque, New Mexico, April 29, 1991. 


\section{Appendix}

\section{Intermittent Transition Flight Data Analysis}

The reentry vehicle used as a source for the flight data used in this investigation was equipped with a total of five photodiode transition indicators. One of the two photodiodes located near the aft end of the vehicle produced an unusual signal which has provided an opportunity to compare analysis results with flight data for what appears to be an intermittent transition phenomenon. Figure Al compares the photodiode signal with the temperature signal measured with a nearsurface thermocouple located next to the photodiode. The photodiode signal exhibits the rapid signal rise to saturation associated with boundary layer transition, but then unsaturates briefly before returning to a saturated level. While it is possible that this drop from saturation was the result of an erroneous measurement, the near-surface thermocouple also produced an unusual signal. The slope of the temperature curve rapidly increased, indicating the increased heating rate associated with boundary layer transition. However, in this case, the slope of the temperature curve briefly returned to a value more closely associated with laminar heating, before again increasing to a value typical of a turbulent heating rate. Thus both the thermocouple and the photodiode signals indicate that the boundary layer at this point on the reentry vehicle transitioned to turbulent flow briefly, then relaminarized briefly before transitioning again to turbulent flow.

Although the cause of this unusual behavior is not known, this data provides an opportunity to apply the analysis techniques presented in this report to an intermittent transition flowfield. Figure A2 presents the cold-wall heating rates which were applied to the reentry vehicle at this station. Laminar heating rates were applied up through a time of $14.4 \mathrm{sec}$, turbulent heating rates were applied from a time of $14.5 \mathrm{sec}$ through a time of $14.7 \mathrm{sec}$, laminar heating rates were applied from a time of $14.8 \mathrm{sec}$ through a time of $14.9 \mathrm{sec}$, and turbulent heating rates were applied from a time of $15.0 \mathrm{sec}$ through the remainder of the flight. The results of the radiating heatshield material analysis using this heating rate history are shown in Figure A3. The flight data in this figure is presented as a series of single points rather than a continuous line. Although this heating rate history was chosen iteratively based on analysis results using the thermocouple data as a guide, it can be seen that the radiating heatshield material analysis tracked the photodiode flight data reasonably well, indicating that intermittent transition can be credited with the unusual flight data, and adding further support to the belief that blackbody radiation from the heatshield material surrounding the quartz window is the source of the photodiode signal. 


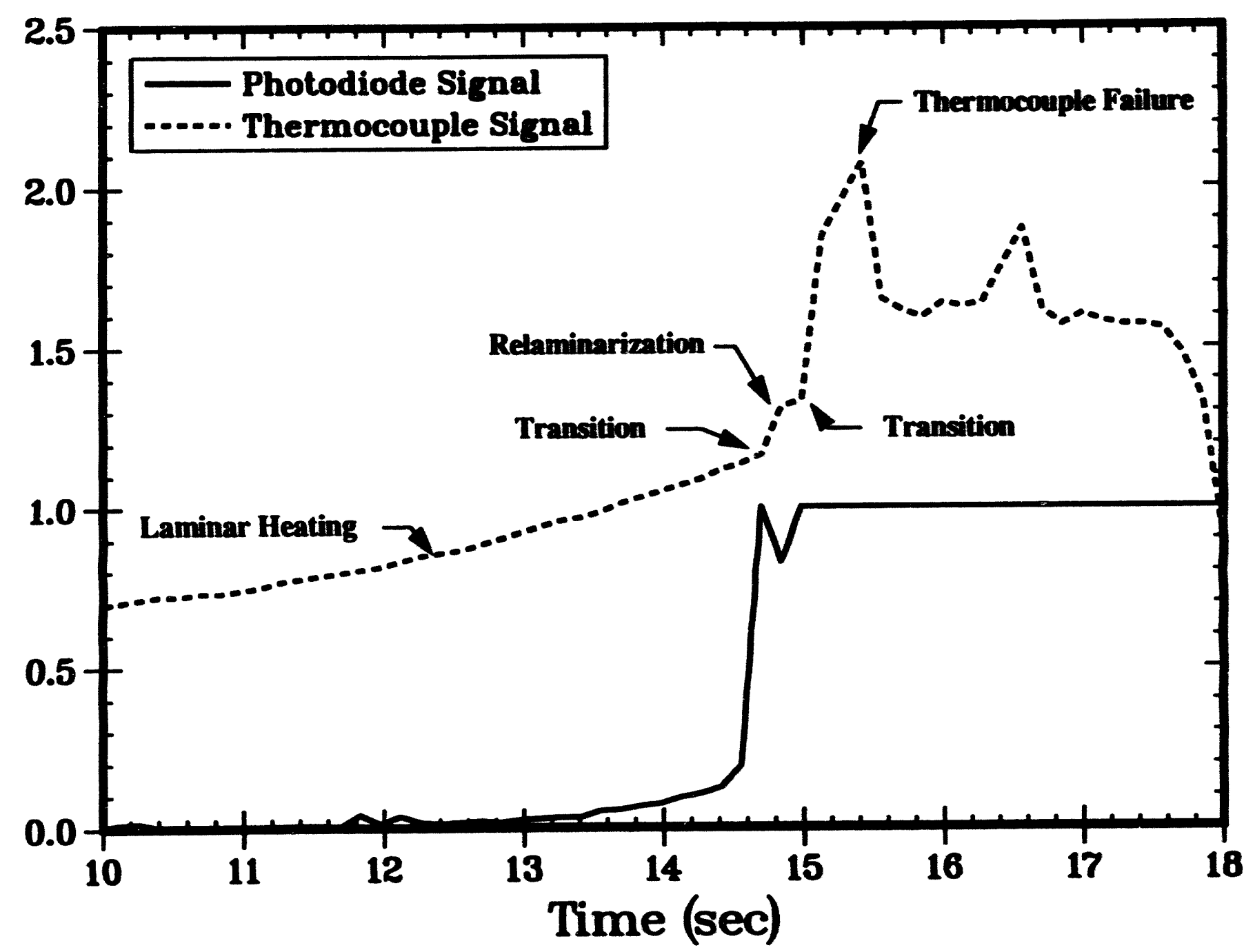

Figure A1: Co-located photodiode and near-surface thermocouple indicating intermittent transition. 


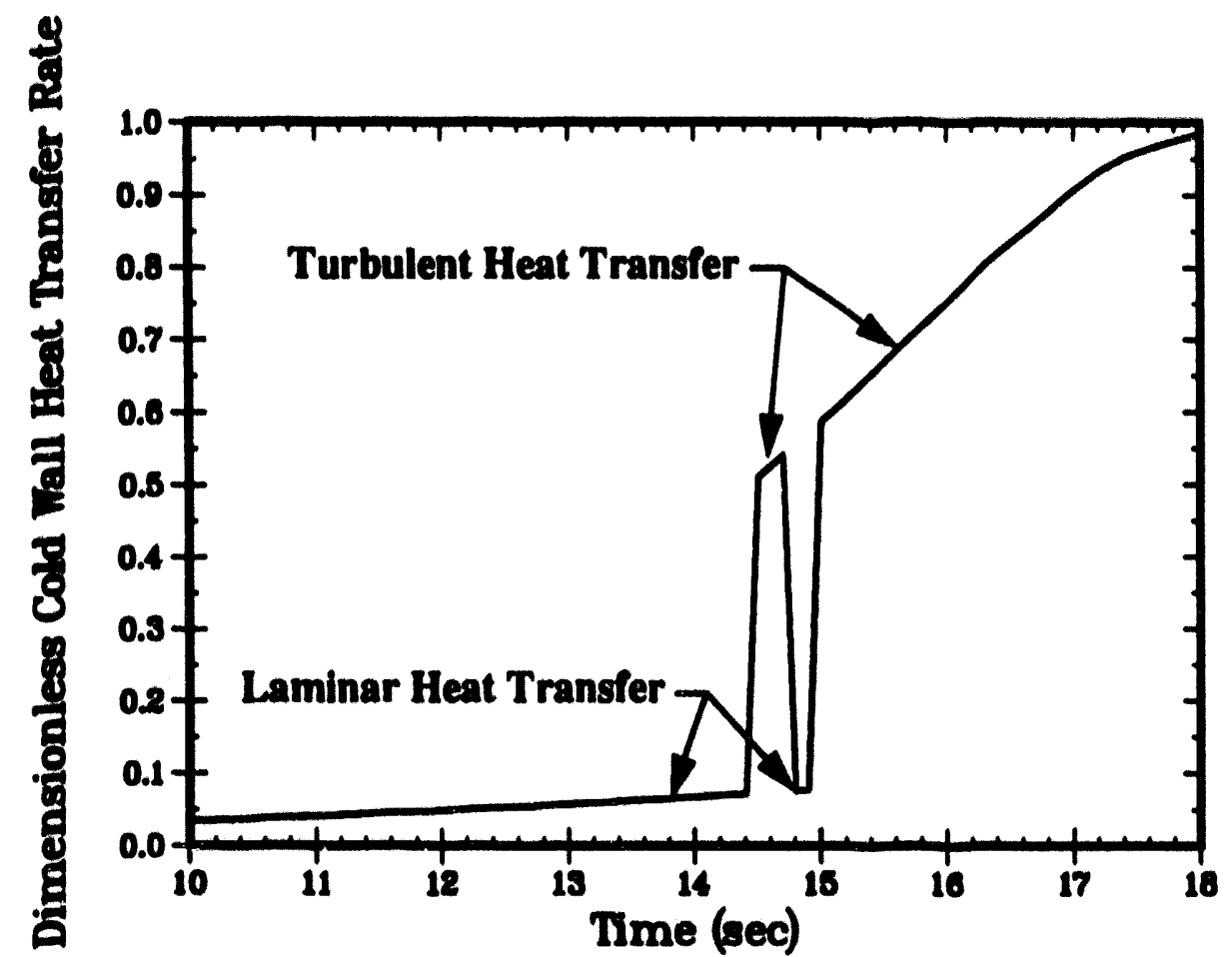

Figure A2: Cold wall heat transfer rate used to simulate intermittent transition.

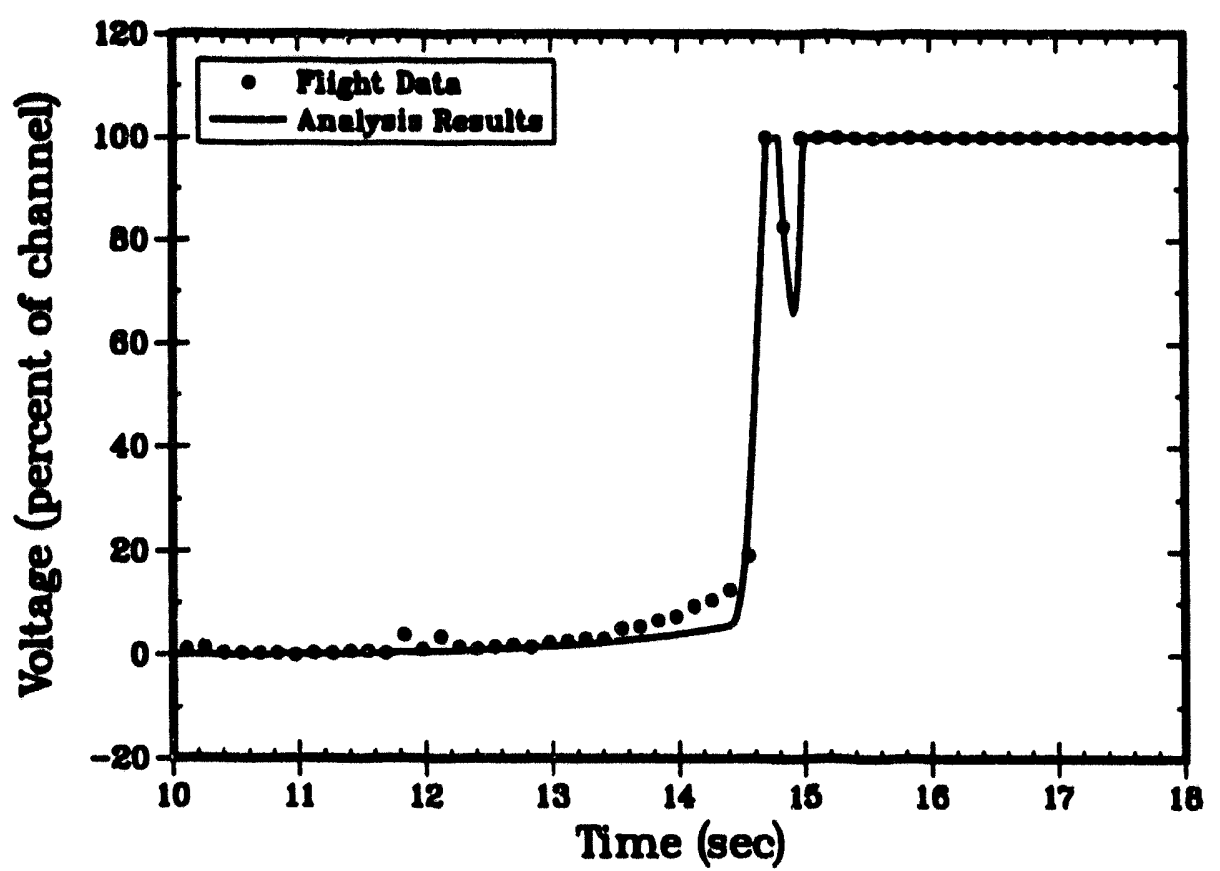

Figure A3: Comparison of flight data and analysis results for the intermittent transition case. 
-

\&

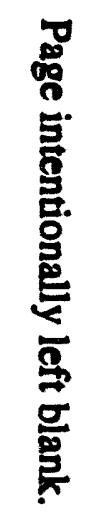

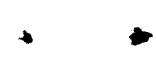

. 


\section{Distribution}

$\begin{array}{lll}\text { MS 0320 } & 01011 & \text { L. M. Lopez } \\ \text { MS 0841 } & 01500 & \text { D. J. McCloskey (Attn. 01512, 01513, 01551, 01552) } \\ \text { MS 0836 } & 01501 & \text { C. W. Peterson } \\ \text { MS 0827 } & 01511 & \text { J. S. Rottler } \\ \text { MS 0443 } & 01561 & \text { H. S. Morgan } \\ \text { MS 0437 } & 01562 & \text { R. K. Thomas } \\ \text { MS 0826 } & 01553 & \text { W. L. Hermina (Attn. Staff) } \\ \text { MS 0826 } & 01553 & \text { D. W. Kuntz (10) } \\ \text { MS 0825 } & 01554 & \text { W. H. Rutledge (Attn. Staff) } \\ \text { MS 0825 } & 01554 & \text { J. L. Payne (5) } \\ \text { MS 0899 } & 07141 & \text { S. A. Landenberger (5) } \\ \text { MS 0619 } & 07151 & \text { G. C. Claycomb } \\ \text { MS 0100 } & 07613-2 & \text { Document Processing for DOE/OSTI (10) } \\ \text { MS 9018 } & 08523 & \text { Central Technical Files } \\ \text { MS 0314 } & 09811 & \text { A. C. Wilken (5) }\end{array}$



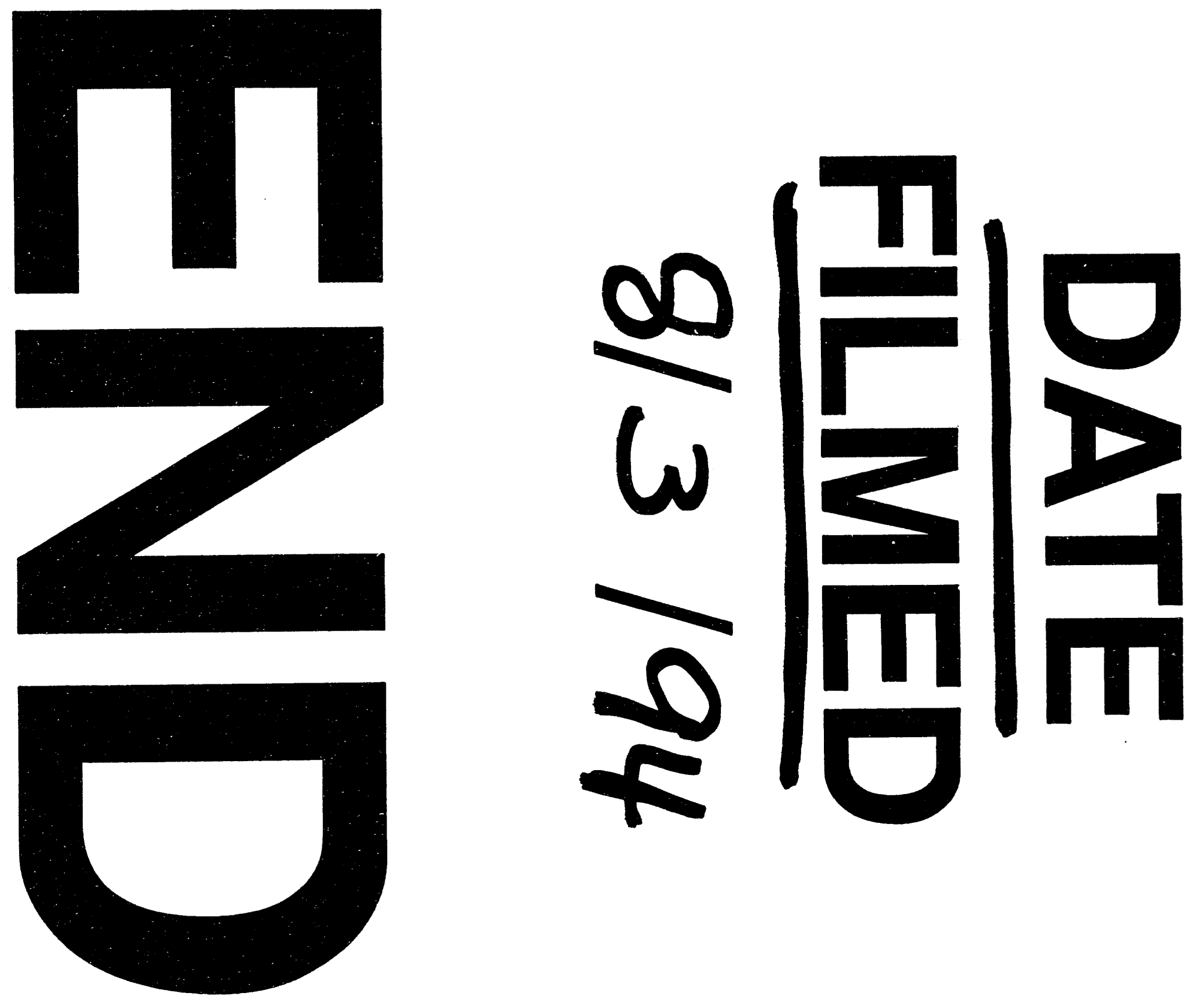
